TRANSACTIONS OF THE

AMERICAN MATHEMATICAL SOCIETY

Volume 365, Number 1, January 2013, Pages 361-407

S 0002-9947(2012)05621-2

Article electronically published on August 10, 2012

\title{
PRIMITIVE SPHERICAL SYSTEMS
}

\author{
P. BRAVI
}

\begin{abstract}
A spherical system is a combinatorial object, arising in the theory of wonderful varieties, defined in terms of a root system. All spherical systems can be obtained by means of some general combinatorial procedures (such as parabolic induction and wonderful fiber product) from the so-called primitive spherical systems. Here we classify the primitive spherical systems. As an application, we prove that the quotients of a spherical system are in correspondence with the so-called distinguished subsets of colors.
\end{abstract}

\section{INTRODUCTION}

Let $G$ be a semisimple group over the complex numbers. A wonderful $G$ variety is a smooth projective quasi-homogeneous $G$-variety such that the irreducible components of the $G$-boundary are quasi-homogeneous of codimension 1 and have nonempty transversal intersection; see [Lu01. Well-known examples are the DeConcini-Procesi complete symmetric varieties.

Wonderful $G$-varieties are spherical, i.e. contain an open orbit for a Borel subgroup of $G$. Moreover, they dominate all the complete simple (i.e. with a unique closed $G$-orbit) spherical varieties. They thus play a central role in the theory of spherical varieties and, in particular, by a result of D. Luna ([Lu01] the classification of wonderful varieties yields the classification of all spherical varieties.

Spherical $G$-systems are combinatorial objects defined axiomatically in terms of the root system of $G$. To each wonderful $G$-variety one can associate a combinatorial invariant which satisfies the axioms of spherical $G$-systems. The Luna conjecture states that wonderful $G$-varieties are classified by spherical $G$-systems, Lu01.

Luna's approach to the classification of wonderful $G$-varieties has led to a proof of the conjecture under special hypotheses: if $G$ has a simply-laced not necessarily connected Dynkin diagram, [Lu01, P03, BP05, Br07, or if all the isotropy groups of the wonderful variety are self-normalizing, BCF08. This proof is constructive, it consists in reducing to some primitive spherical systems and checking case-bycase that all primitive spherical systems correspond to one and only one wonderful variety.

This approach actually works in general for any group $G$ and a complete proof of the conjecture using this method is an on-going project (see BP11]).

Besides this, I. Losev has extended the uniqueness part of the proof in full generality essentially avoiding case-by-case considerations, [Lo09]. Furthermore, invariant Hilbert schemes and equivariant deformation theory provide another approach to the proof of the conjecture; see [BCF10] and also [CF08, CF09].

Received by the editors July 19, 2010 and, in revised form, January 21, 2011 and April 4, 2011. 2010 Mathematics Subject Classification. Primary 14M27; Secondary 05E10, 05E15. Key words and phrases. Wonderful varieties, root systems. 
In this paper we obtain the complete list of primitive spherical systems for any group $G$. This gives an account on the combinatorial structure of all spherical systems.

Some general properties of spherical systems can actually be proved by reducing to the primitive spherical systems. For instance, we prove in this way that all the so-called distinguished subsets of colors are good, that is, give rise to well-defined quotient spherical systems. This technical property was implicitly conjectured by Luna as well; see Lu01, Remarque 3.3].

An important feature of the theory is the interplay of geometry, algebraic group theory and combinatorics: many properties of a wonderful variety or of its isotropy groups can be characterized in combinatorial terms on the corresponding spherical system; see [Lu01, BL11, BP11.

This paper concerns only the combinatorial aspects of spherical systems. It is self-contained and only a basic knowledge of root systems is assumed. Most of the combinatorial constructions here in use are actually motivated by their corresponding geometric or group-theoretic counterpart: for these aspects of the theory we refer in particular to BP11.

In Section 1 we recall the definition of a spherical system and of the related basic notions. In Section 2 we give the definition of a primitive spherical system and of a spherical system with a primitive positive 1-comb. We provide the list of primitive spherical systems and of spherical systems with a primitive positive 1-comb, and we prove that such lists are complete. In Section 3 we prove that all the distinguished subsets of colors are good. In the appendix we list all the spherical systems of rank $\leq 2$, with their minimal quotients.

To prove the Luna conjecture one can assume without loss of generality that the group $G$ is of adjoint type, i.e. with trivial center. We will make this assumption. This implies that all the so-called spherical roots lie in the root lattice of $G$; see Definition 1.1 .

Without referring to the group $G$, spherical $G$-systems will just be called spherical $R$-systems, where $R$ is the root system of $G$.

The list presented here includes some already known classes of primitive spherical $R$-systems: those with $R$ with irreducible components of type A, D or E ([Lu01, BP05, Br07]), of type A or C ([P03]), of type F ([BL11] ) or the so-called strict primitive spherical systems ([BCF10] $)$. About this, it is necessary to remark that our present definition of a primitive spherical system is slightly more restrictive than that of the previous papers (BP05, Br07, BCF10]); see Definition 2.9.

The author is indebted to D. Luna and G. Pezzini for their help and support.

\section{BASIC DEFINITIONS}

In this section we recall the basic definitions concerning the spherical systems; general references are Lu01 and BL11.

1.1. Spherical systems. Let $R$ be a reduced root system, with scalar product $\langle$,$\rangle . Let S$ be a set of simple roots of $R$. For all $\alpha \in S$, the corresponding simple coroot is denoted by $\alpha^{\vee}=2 \alpha /\langle\alpha, \alpha\rangle$. The simple roots of an irreducible root system will be labeled as in $\mathrm{Bo}$.

Letting $\sigma$ be an element of $\mathbb{N} S$, say $\sigma=\sum_{\alpha \in S} n_{\alpha} \alpha$, define the support of $\sigma$ denoted by $\operatorname{supp} \sigma$ to be the subset of simple roots $\alpha$ such that $n_{\alpha} \neq 0$. 
Definition 1.1. The set of spherical $R$-roots, denoted by $\Sigma(R)$, is the set of $\sigma \in \mathbb{N} S$ such that 1

- $\sigma=\alpha+\beta$, where $\alpha$ and $\beta$ are orthogonal simple roots ( $\sigma$ is said to be of type $a a)$,

- or $\operatorname{supp} \sigma$ is the set of simple roots of an irreducible root subsystem of $R$ and, after restricting $S$ to $\operatorname{supp} \sigma, \sigma$ is one of the following:

\begin{tabular}{lll} 
type of supp & $\sigma$ & type of $\sigma$ \\
\hline $\mathrm{A}_{n}, n \geq 1$ & $\sum_{i=1}^{n} \alpha_{i}$ & $a(n)$ \\
$\mathrm{A}_{1}$ & $2 \alpha_{1}$ & $2 a$ \\
$\mathrm{~B}_{n}, n \geq 2$ & $\sum_{i=1}^{n} \alpha_{i}$ & $b(n)$ \\
& $\sum_{i=1}^{n} 2 \alpha_{i}$ & $2 b(n)$ \\
$\mathrm{B}_{3}$ & $\alpha_{1}+2 \alpha_{2}+3 \alpha_{3}$ & \\
$\mathrm{C}_{n}, n \geq 3$ & $\alpha_{1}+\left(\sum_{i=2}^{n-1} 2 \alpha_{i}\right)+\alpha_{n}$ & $c(n)$ \\
$\mathrm{D}_{n}, n \geq 3$ & $\left(\sum_{i=1}^{n-2} 2 \alpha_{i}\right)+\alpha_{n-1}+\alpha_{n}$ & $d(n)$ \\
$\mathrm{F}_{4}$ & $\alpha_{1}+2 \alpha_{2}+3 \alpha_{3}+2 \alpha_{4}$ & $f$ \\
$\mathrm{G}_{2}$ & $2 \alpha_{1}+\alpha_{2}$ & $g$ \\
& $4 \alpha_{1}+2 \alpha_{2}$ & $2 g$ \\
& $\alpha_{1}+\alpha_{2}$ &
\end{tabular}

We also set $d(2)=a a, b(1)=a(1), 2 b(1)=2 a, c(2)=b(2)$.

Definition 1.2. Let $\sigma$ be a spherical $R$-root. Define $S^{p}(\sigma)$ to be the set of simple roots orthogonal to $\sigma$ and $S^{p p}(\sigma)$ to be equal to

- $S^{p}(\sigma) \cap \operatorname{supp} \sigma \backslash\left\{\alpha_{n}\right\}$ if $\sigma=\sum_{i=1}^{n} \alpha_{i}$ with support of type $\mathrm{B}_{n}$,

- $S^{p}(\sigma) \cap \operatorname{supp} \sigma \backslash\left\{\alpha_{1}\right\}$ if $\sigma$ has support of type $\mathrm{C}_{n}$,

- $S^{p}(\sigma) \cap \operatorname{supp} \sigma$ otherwise.

Let $S^{p}$ be a subset of $S$. Then $S^{p}$ and $\sigma$ are said to be compatible if

$$
S^{p p}(\sigma) \subset S^{p} \subset S^{p}(\sigma) .
$$

Definition 1.3. A triple $\mathscr{S}=\left(S^{p}, \Sigma, A\right)$, where

- $S^{p} \subset S$

- $\Sigma \subset \Sigma(R)$ without proportional elements

- $A$ is a finite set endowed with a pairing $c: A \times \Sigma \rightarrow \mathbb{Z}$

is called a spherical $R$-system if the following axioms are satisfied. For all $\alpha \in S \cap \Sigma$, set $A(\alpha)=\{D \in A: c(D, \alpha)=1\}$.

(A1) For all $D \in A$ and $\sigma \in \Sigma, c(D, \sigma) \leq 1$, and if $c(D, \sigma)=1$, then $\sigma \in S$.

(A2) For all $\alpha \in S \cap \Sigma$, $\operatorname{card}(A(\alpha))=2$, and if $A(\alpha)=\left\{D_{\alpha}^{+}, D_{\alpha}^{-}\right\}$, then $c\left(D_{\alpha}^{+}, \sigma\right)+c\left(D_{\alpha}^{-}, \sigma\right)=\left\langle\alpha^{\vee}, \sigma\right\rangle$ for all $\sigma \in \Sigma$.

(A3) $A=\bigcup_{\alpha \in S \cap \Sigma} A(\alpha)$.

( $\Sigma 1)$ If $2 \alpha \in 2 S \cap \Sigma$, then $\frac{1}{2}\left\langle\alpha^{\vee}, \sigma\right\rangle \in \mathbb{Z}_{\leq 0}$ for all $\sigma \in \Sigma \backslash\{2 \alpha\}$.

( $\Sigma 2)$ If $\alpha$ and $\beta$ are orthogonal simple roots with $\alpha+\beta \in \Sigma$, then $\left\langle\alpha^{\vee}, \sigma\right\rangle=$ $\left\langle\beta^{\vee}, \sigma\right\rangle$ for all $\sigma \in \Sigma$.

(S) $S^{p}$ and $\sigma$ are compatible for all $\sigma \in \Sigma$.

\footnotetext{
${ }^{1}$ Notice that this definition is slightly more restrictive than that of [Lu01] where a spherical root does not necessarily belong to $\mathbb{N} S$. However, this holds if the acting group $G$ is assumed to be of adjoint type.
} 
In the following, let $\mathscr{S}=\left(S^{p}, \Sigma, A\right)$ be a spherical $R$-system.

Let us fix some terminology: the spherical roots of $\mathscr{S}$ are the elements of $\Sigma$; the rank of $\mathscr{S}$ is the cardinality of $\Sigma$; the restricted Cartan pairing of $\mathscr{S}$ is the pairing $c: A \times \Sigma \rightarrow \mathbb{Z}$.

Definition 1.4. Set

- $S^{a}=S \cap \Sigma$,

- $S^{2 a}=2 S \cap \Sigma$,

- $S^{b}=S \backslash\left(S^{p} \cup S^{a} \cup S^{2 a}\right)$.

The set of colors of $\mathscr{S}$ denoted by $\Delta$ is a finite set, the disjoint union of $\Delta^{a}, \Delta^{2 a}$ and $\Delta^{b}$,

- $\Delta^{a}=A$,

- $\Delta^{2 a}=\left\{D_{2 \alpha}: \alpha \in S^{2 a}\right\}$,

- $\Delta^{b}=\left\{D_{\alpha}: \alpha \in S^{b}\right\}$, where $D_{\alpha}=D_{\beta}$ if and only if $\alpha \perp \beta$ and $\alpha+\beta \in \Sigma$.

The set of colors $\Delta$ of $\mathscr{S}$ is endowed with a pairing $c: \Delta \times \Sigma \rightarrow \mathbb{Z}$ (the Cartan pairing) extending the restricted Cartan pairing as follows:

- $c\left(D_{2 \alpha}, \sigma\right)=\frac{1}{2}\left\langle\alpha^{\vee}, \sigma\right\rangle$ for all $\alpha \in S^{2 a}$ and $\sigma \in \Sigma$,

- $c\left(D_{\alpha}, \sigma\right)=\left\langle\alpha^{\vee}, \sigma\right\rangle$ for all $\alpha \in S^{b}$ and $\sigma \in \Sigma$.

Furthermore, set $\Delta(\alpha)=A(\alpha)$ if $\alpha \in S^{a}, \Delta(\alpha)=\left\{D_{2 \alpha}\right\}$ if $\alpha \in S^{2 a}$ and $\Delta(\alpha)=$ $\left\{D_{\alpha}\right\}$ if $\alpha \in S^{b}$.

Definition 1.5. The difference between the cardinality of $\Delta$ and the cardinality of $\Sigma$ (the rank of $\mathscr{S})$ is called the defect of $\mathscr{S}$ and denoted by $\mathrm{d}(\mathscr{S})$.

Remark 1.6. From the classification of rank 2 spherical systems (see the appendix or [W96]) it turns out that all the pairs of spherical roots of any spherical system have nonpositive scalar product. This implies that the spherical roots of any spherical system are linearly independent 2 This implies also that $\Sigma$ is a basis of a reduced root system.

The Cartan pairing can be extended by $\mathbb{Z}$-linearity to $c: \mathbb{Z} \Delta \times \mathbb{Z} \Sigma \rightarrow \mathbb{Z}$.

\subsection{Localization.}

Definition 1.7. Let $\mathscr{S}=\left(S^{p}, \Sigma, A\right)$ be a spherical $R$-system. Let $S^{\prime}$ be a subset of $S$ and $R^{\prime}$ the corresponding root subsystem of $R$. The spherical $R^{\prime}$-system obtained from $\mathscr{S}$ by localization is $\mathscr{S}^{\prime}=\left(\left(S^{\prime}\right)^{p}, \Sigma^{\prime}, A^{\prime}\right)$ :

- $\left(S^{\prime}\right)^{p}=S^{p} \cap S^{\prime}$

- $\Sigma^{\prime}=\left\{\sigma \in \Sigma: \operatorname{supp} \sigma \subset S^{\prime}\right\}$,

- $A^{\prime}=\bigcup A(\alpha)$ for all $\alpha \in \Sigma \cap S^{\prime}$.

Definition 1.8. A spherical $R$-system $\mathscr{S}=\left(S^{p}, \Sigma, A\right)$ is called cuspidal if

$$
\bigcup_{\sigma \in \Sigma} \operatorname{supp} \sigma=S
$$

\footnotetext{
${ }^{2}$ We thank A. Maffei for having pointed out to us this easy argument.
} 
1.3. Quotients. Let $\mathscr{S}=\left(S^{p}, \Sigma, A\right)$ be a spherical $R$-system with set of colors $\Delta$. Definition 1.9. An element $D$ of $\mathbb{Z} \Delta$ is called positive if $c(D, \sigma) \geq 0$ for all $\sigma \in \Sigma$.

Definition 1.10. A subset $\Delta^{\prime} \subset \Delta$ of colors of $\mathscr{S}$ is called distinguished if there exists a positive element in $\mathbb{N}_{>0} \Delta^{\prime}$.

Definition 1.11. Let $\Delta^{\prime} \subset \Delta$ be a distinguished subset of colors of $\mathscr{S}$. Consider $S^{p} / \Delta^{\prime}=\left\{\alpha \in S: \Delta(\alpha) \subset \Delta^{\prime}\right\}$, the set $\Sigma / \Delta^{\prime}$ of minimal generators of the semigroup

$$
\left\{\sigma \in \mathbb{N} \Sigma: c(D, \sigma)=0 \forall D \in \Delta^{\prime}\right\}
$$

and the subset $A / \Delta^{\prime}$ of $A$ given by $S \cap \Sigma / \Delta^{\prime}$. Define

$$
\mathscr{S} / \Delta^{\prime}=\left(S^{p} / \Delta^{\prime}, \Sigma / \Delta^{\prime}, A / \Delta^{\prime}\right) .
$$

A distinguished subset $\Delta^{\prime} \subset \Delta$ is called good if $\mathscr{S} / \Delta^{\prime}$ is a spherical $R$-system. In this case $\mathscr{S} / \Delta^{\prime}$ is called a quotient spherical $R$-system.

The set of colors of the quotient spherical system $\mathscr{S} / \Delta^{\prime}$ can be identified with $\Delta \backslash \Delta^{\prime}$.

\section{Definition 1.12.}

- A good distinguished subset $\Delta^{\prime}$ is called homogeneous if $\Sigma / \Delta^{\prime}=\emptyset$.

- A quotient of a spherical system given by a minimal good distinguished subset of colors is called minimal.

1.4. Luna diagrams. Let us recall how to visualize a spherical system $\mathscr{S}=\left(S^{p}, \Sigma, A\right)$ via its Luna diagram.

The spherical roots are represented on the Dynkin diagram of their support as in Table 1 .

Additional not shadowed circles around vertices may occur: $S^{p}$ equals the set of vertices having no circles around, below or above.

The set $S \cap \Sigma$ corresponds to the set of vertices which have circles above and below. For each $\alpha \in S \cap \Sigma$, these two circles are identified with the elements of $A(\alpha)$, the circle above to $D_{\alpha}^{+}$, where $D_{\alpha}^{+}$is such that $c\left(D_{\alpha}^{+}, \sigma\right) \in\{1,0-1\}$, for every spherical root $\sigma$. Circles in different $A(\alpha)$ 's are joined by a line if they correspond to the same element in $A$. Finally, for every spherical root $\sigma$ not orthogonal to $\alpha$ such that $c\left(D_{\alpha}^{+}, \sigma\right)=-1$, there is an arrow (usually only a symbol "<" or ">"), starting from the circle corresponding to $D_{\alpha}^{+}$and pointing toward $\sigma$. The set $A$ and the restricted Cartan pairing $c: A \times \Sigma \rightarrow \mathbb{Z}$ can then be recovered by Axiom A2.

Arrows between diagrams. An arrow from the diagram of $\mathscr{S}$ to the diagram of $\mathscr{S}^{\prime}$ means that the spherical system $\mathscr{S}$ has $\mathscr{S}^{\prime}$ as quotient spherical system. A minimal quotient with decreasing defect is denoted by a dashed arrow; a minimal quotient with nondecreasing defect is denoted by a continuous arrow.

1.5. Spherical systems of rank $\leq 2$. The spherical systems of rank 1 and of rank 2 are known (W96]); their classification can directly be deduced from the axioms of the spherical systems.

In the appendix we recall the Luna diagrams of all the cuspidal spherical systems of rank 1 and of all the cuspidal spherical systems of rank 2 that are not a direct product of two spherical systems of rank 1 (see the appendix for a precise definition of direct product). We also provide their minimal quotient spherical systems. 
TABLE 1. Spherical roots

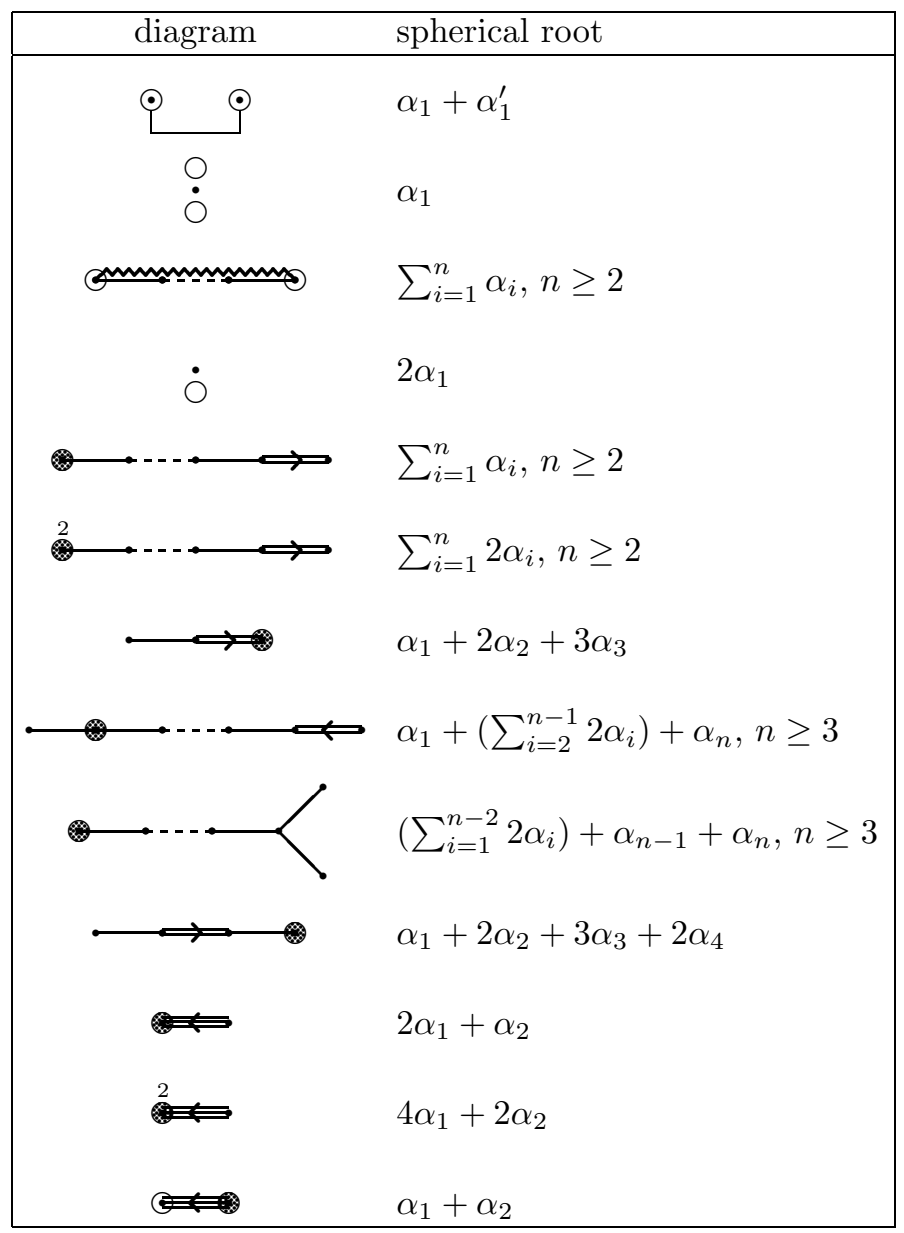

Remark 1.13. All the distinguished subsets of colors of the spherical systems of rank $\leq 2$ are good.

\section{Classification of SPHerical Systems}

2.1. Primitive spherical systems. Here we introduce the notion of a primitive spherical $R$-system and give the list of all of them, for any $R$. We need to start with the definition of three preliminary notions: decomposition, positive comb and tail.

Let $\mathscr{S}=\left(S^{p}, \Sigma, A\right)$ be a spherical $R$-system.

Definition 2.1 (Decomposition). Let $\Delta^{\prime}$ and $\Delta^{\prime \prime}$ be good distinguished subsets of colors of $\mathscr{S}$ such that

- $\left(S^{p} / \Delta^{\prime} \backslash S^{p}\right) \perp\left(S^{p} / \Delta^{\prime \prime} \backslash S^{p}\right)$ and

- $\Sigma \subset\left(\Sigma / \Delta^{\prime} \cup \Sigma / \Delta^{\prime \prime}\right)$. 
Then we say that $\Delta^{\prime}$ and $\Delta^{\prime \prime}$ decompose $\mathscr{S} 3$ and that $\mathscr{S}$ is a wonderful fiber product of $\mathscr{S} / \Delta^{\prime}$ and $\mathscr{S} / \Delta^{\prime \prime}$ over $\mathscr{S} /\left(\Delta^{\prime} \cup \Delta^{\prime \prime}\right)$.

Example 2.2. Let us consider the spherical systems with the following diagrams:

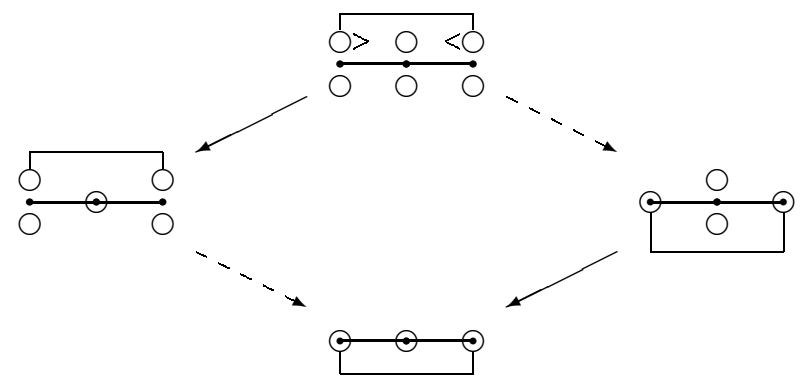

Let $\mathscr{S}$ be the spherical system corresponding to the diagram on the top of the picture. Let $\Delta^{\prime}=\left\{D_{\alpha_{2}}^{+}\right\}$and $\Delta^{\prime \prime}=\left\{D_{\alpha_{1}}^{-}, D_{\alpha_{3}}^{-}\right\}$, subsets of colors of $\mathscr{S}$. Then $\mathscr{S}$ is a wonderful fiber product of $\mathscr{S} / \Delta^{\prime}$ and $\mathscr{S} / \Delta^{\prime \prime}$ over $\mathscr{S} /\left(\Delta^{\prime} \cup \Delta^{\prime \prime}\right)$.

Definition 2.3 (Positive comb). A positive color $D \in A$ (see Definition 1.9) is called a positive comb, or a positive $n$-comb, where $n$ equals the cardinality of $S_{D}=\{\alpha \in S: D \in \Delta(\alpha)\}$.

Let $n$ be the cardinality of $S$. By abuse of terminology a spherical system with $\Sigma=S$ and a positive $n$-comb $D$ with $S_{D}=S$ is often called an $n$-comb.

Example 2.4. The following has a positive 2-comb:

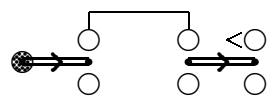

Definition 2.5 (Tail of classical type). Let $\sigma \in \Sigma$, with supp $\sigma$ included in the set of simple roots $\alpha_{1}, \ldots, \alpha_{n}$ of an irreducible component $R^{\prime}$ of $R$. Suppose that there exists a good distinguished subset of colors $\Delta^{\prime}$ such that $\Sigma / \Delta^{\prime}=\{\sigma\}$. Then $\sigma$ is called:

- tail of type $b(m), 1 \leq m \leq n$, if $R^{\prime}$ is of type $\mathrm{B}_{n}, \sigma=\alpha_{n-m+1}+\ldots+\alpha_{n}$ and $\alpha_{n} \in S^{p}$ if $m>1$ (or $c\left(D_{\alpha_{n}}^{+}, \sigma^{\prime}\right)=c\left(D_{\alpha_{n}}^{-}, \sigma^{\prime}\right)$ for all $\sigma^{\prime} \in \Sigma$ if $m=1$ );

- tail of type $2 b(m), 1 \leq m \leq n$, if $R^{\prime}$ is of type $\mathrm{B}_{n}$ and $\sigma=2 \alpha_{n-m+1}+\ldots+$ $2 \alpha_{n}$

- tail of type $c(m), 2 \leq m \leq n$, if $R^{\prime}$ is of type $C_{n}$ and $\sigma=\alpha_{n-m+1}+$ $2 \alpha_{n-m+2}+\ldots+2 \alpha_{n-1}+\alpha_{n}$

- tail of type $d(m), 2 \leq m \leq n$, if $R^{\prime}$ is of type $\mathrm{D}_{n}$ and $\sigma=2 \alpha_{n-m+1}+\ldots+$ $2 \alpha_{n-2}+\alpha_{n-1}+\alpha_{n}$.

Example 2.6. Here is an example of each of the above types of tails, with $R^{\prime}=R$ and $\Delta^{\prime}=\left\{D_{\alpha_{1}}\right\}$.

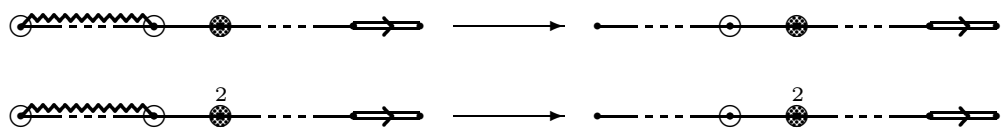

\footnotetext{
${ }^{3}$ This definition is slightly different from that given in the previous papers ( $\left.\left.\mathrm{Lu} 01, \mathrm{BP} 05, \mathrm{Br} 07\right]\right)$, but actually equivalent.
} 


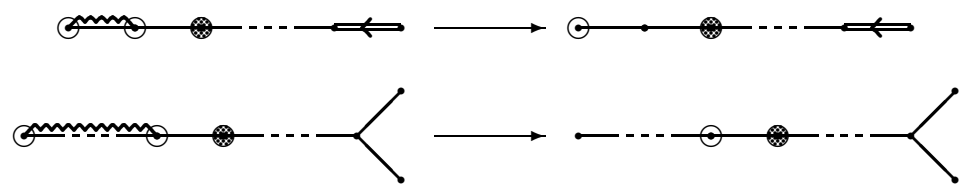

Definition 2.7 (Tail of exceptional type). Let $\widetilde{\Sigma}=\left\{\sigma_{1}, \sigma_{2}\right\} \subset \Sigma$, with supp $\sigma_{1} \cup$ $\operatorname{supp} \sigma_{2}$ included in the set of simple roots $\alpha_{1}, \ldots, \alpha_{n}$ of an irreducible component $R^{\prime}$ of $R$. Suppose that there exists a good distinguished subset of colors $\Delta^{\prime}$ such that $\Sigma / \Delta^{\prime}=\widetilde{\Sigma}$. Then $\widetilde{\Sigma}$ is called:

- tail of type $(a a, a a)$ if $R^{\prime}$ is of type $\mathrm{E}_{6}$ and $\widetilde{\Sigma}=\left\{\alpha_{1}+\alpha_{6}, \alpha_{3}+\alpha_{5}\right\}$;

- tail of type $(d 3, d 3)$ if $R^{\prime}$ is of type $\mathrm{E}_{7}$ and $\widetilde{\Sigma}=\left\{\alpha_{2}+2 \alpha_{4}+\alpha_{5}, \alpha_{5}+2 \alpha_{6}+\alpha_{7}\right\}$;

- tail of type $(d 5, d 5)$ if $R^{\prime}$ is of type $\mathrm{E}_{8}$ and $\widetilde{\Sigma}=\left\{2 \alpha_{1}+\alpha_{2}+2 \alpha_{3}+2 \alpha_{4}+\right.$ $\left.\alpha_{5}, \alpha_{2}+\alpha_{3}+2 \alpha_{4}+2 \alpha_{5}+2 \alpha_{6}\right\}$

- tail of type $(2 a, 2 a)$ if $R^{\prime}$ is of type $\mathrm{F}_{4}$ and $\widetilde{\Sigma}=\left\{2 \alpha_{3}, 2 \alpha_{4}\right\}$.

Example 2.8. Here is an example of each of the above types of tails, with $R^{\prime}=R$ and $\Delta^{\prime}$ respectively equal to $\left\{D_{\alpha_{2}}\right\},\left\{D_{\alpha_{1}}\right\},\left\{D_{\alpha_{8}}\right\},\left\{D_{\alpha_{1}}\right\}$.

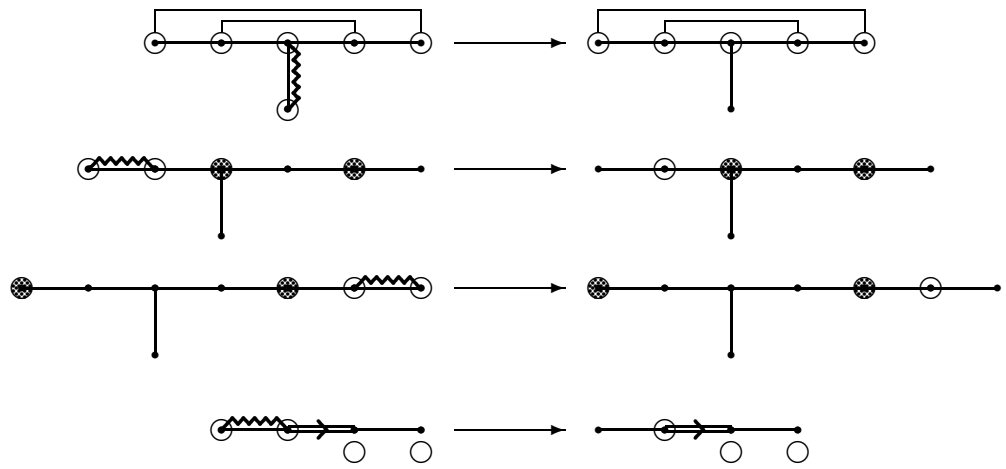

We are now ready to give the definition of a primitive spherical system.

Definition 2.9 (Primitive spherical system). A spherical $R$-system is called primitive if it is cuspidal, not decomposable, without positive combs and without tails (of type $b(m), 2 b(m), c(m), d(m),(a a, a a),(d 3, d 3),(d 5, d 5)$ or $(2 a, 2 a))$ 노

To present them all, we subdivide the primitive spherical systems into clans, which can be characterized as follows:

clan R: primitive spherical systems with a spherical root of type $a a$ or $2 a$ and without spherical roots with overlapping supports or with a spherical root of type $d(m), m \geq 3$;

clan S: primitive spherical systems with only spherical roots of type $a(m)$ $(m \geq 1)$ or $b(2)$ (with $\left.S^{p}=\emptyset\right)$ without spherical roots with overlapping supports;

clan T: primitive spherical systems with a spherical root of type $a(m)(m \geq$ 1) whose support meets the support of another spherical root.

\footnotetext{
${ }^{4}$ Notice that in the previous papers ( $\left.\left.\mathrm{BP} 05, \mathrm{Br} 07, \mathrm{BCF} 10\right]\right)$ this definition was less restrictive: tails were allowed.
} 
We give only the Luna diagram of the spherical systems. We use identifications between spherical roots as in Definition 1.1 namely, when in a diagram there is a spherical root of type $a(m), 2 b(m)$ or $d(m)$ without overlapping support, then $m$ must be intended as $\geq 1, \geq 1$ or $\geq 2$, respectively. In the diagrams of family $c$ of the clan $\mathrm{T}$ there is always a spherical root of type $c(m)$, here $m$ must be intended as $\geq 2$.

Theorem 2.10. The clans $R, S$ and $T$, given below, contain all the primitive spherical $R$-systems of rank $>2$, up to external automorphism of the root system $R$.

In Section 2.5 we prove the above theorem; that is, we show that the list presented here is complete, by explaining step-by-step how it is obtained.

\section{Clan R.}

$\mathbf{R}-\mathbf{1}$.

$\mathrm{a}^{\text {aa }}(p, p)$

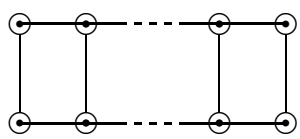

$\mathrm{b}^{\text {aa }}(p, p)$

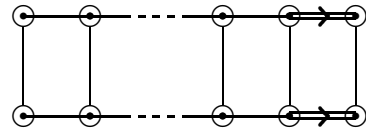

$\mathrm{c}^{\mathrm{aa}}(p, p)$

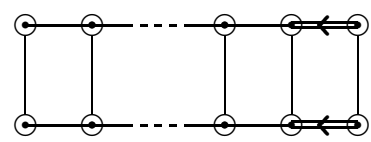

$\mathrm{d}^{\mathrm{aa}}(p, p)$

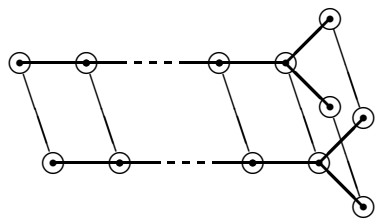

$\mathrm{e}^{\mathrm{aa}}(p, p)$

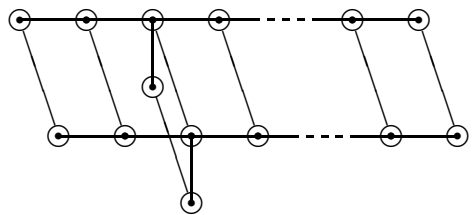

$f^{\text {aa }}(4,4)$

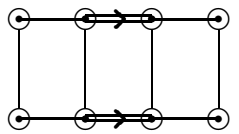

$\mathrm{g}^{\mathrm{aa}}(2,2)$

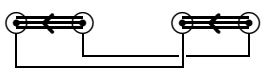


R-2.

$\mathbf{R}-\mathbf{2}^{\prime}$
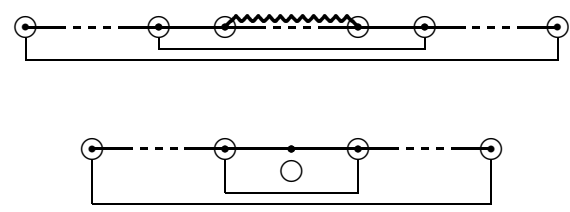

R-3.

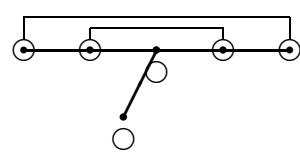

R-4.

$\mathrm{a}^{2 \mathrm{a}}(p)$

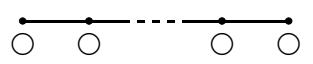

$\mathrm{b}^{2 \mathrm{a}}(p)$

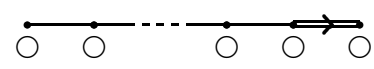

$c^{2 a}(p)$

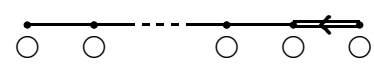

$\mathrm{d}^{2 \mathrm{a}}(p)$

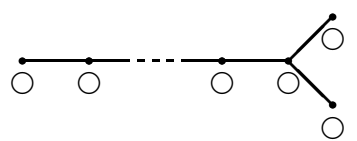

$\mathrm{e}^{2 \mathrm{a}}(p)$

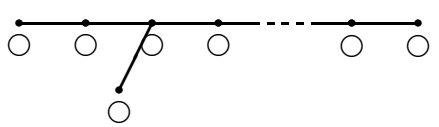

$f^{2 a}(4)$

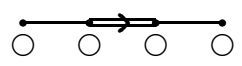

$\mathrm{g}^{2 \mathrm{a}}(2)$

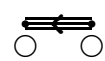

R-5.

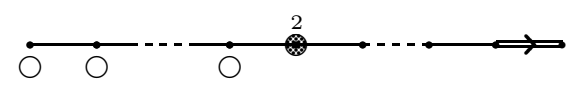

R-6.

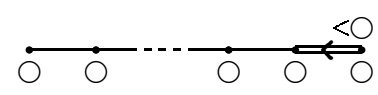

R-7.

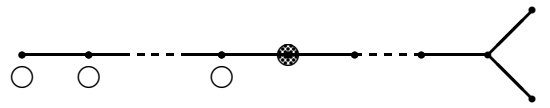

R-8.

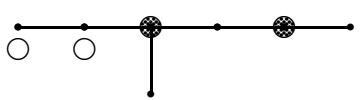


R-9.

R-10.

R-10'.
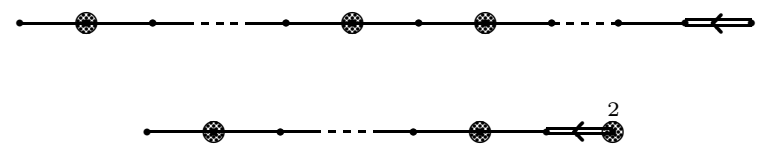

R-11.

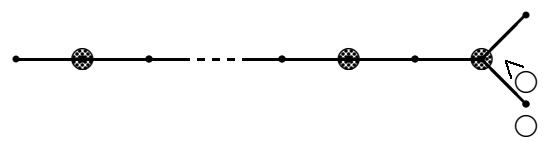

$\mathbf{R}-11^{\prime}$.

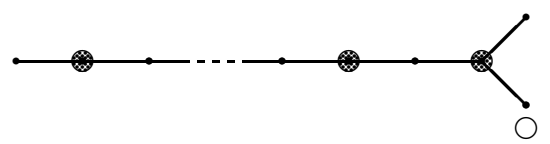

R-12.

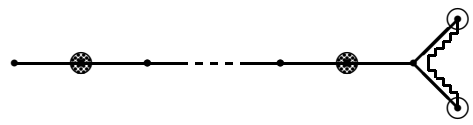

R-13.

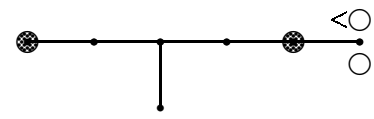

$\mathbf{R}-13^{\prime}$.

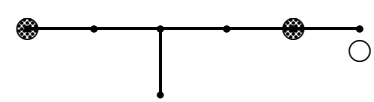

R-14.

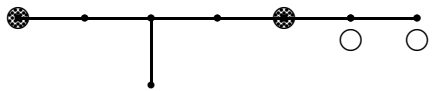

\section{Clan S.}

Family $\mathbf{x}$

S-1. $\operatorname{bd}^{\times}(p, p), p \geq 2\left(\right.$ or $\operatorname{ab}^{\times}(3,3)$ if $p=3, \operatorname{ab}^{\times}(1,1,2)$ if $\left.p=2\right)$

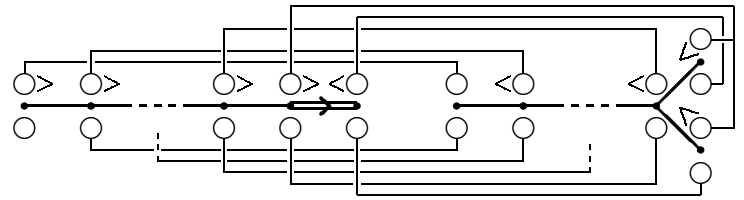

S-2. $b^{\times}(p-1, p), p \geq 2\left(\right.$ or $\mathrm{ab}^{\times}(3,2)$ if $p=3, \mathrm{a}^{\times}(1,1,1)$ if $\left.p=2\right)$

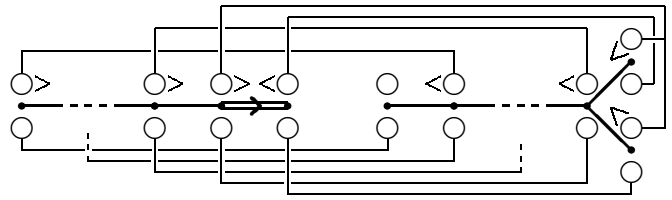


$\mathbf{S}-\mathbf{3} \cdot \mathrm{bc}^{\times}(2,3)$

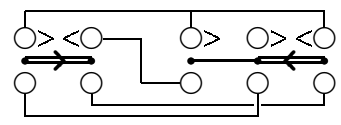

\section{Family y}

S-4. $\mathbf{a}^{\mathrm{y}}(p, p), p \geq 2$

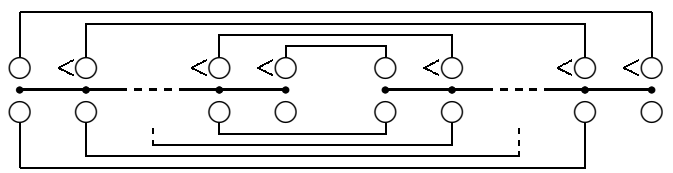

S-5. $\mathrm{a}^{\mathrm{y}}(p-1, p), p \geq 2$

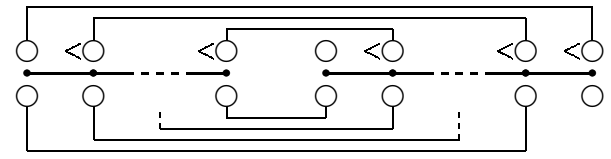

S-6. $\mathrm{ab}^{\mathrm{y}}(p, p), p \geq 2$

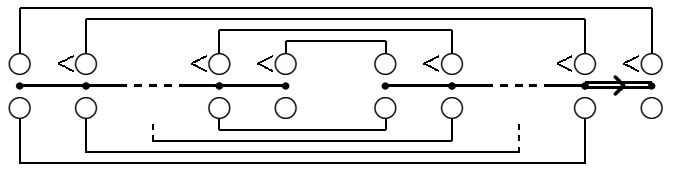

S-7. $\mathrm{ab}^{\mathrm{y}}(p-1, p), p \geq 2$

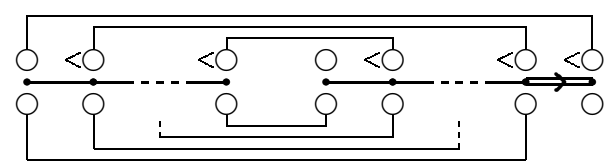

$\mathbf{S}-\mathbf{8} \cdot \mathrm{b}^{\mathrm{y}}(p, p), p \geq 2$

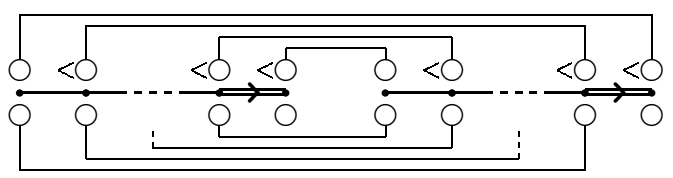

S-9. $\mathrm{d}^{\mathrm{y}}(p+1, p+1), p \geq 3\left(\mathrm{~d}^{\mathrm{y}}(3,3)=a^{\mathrm{z}}(3,3)\right)$

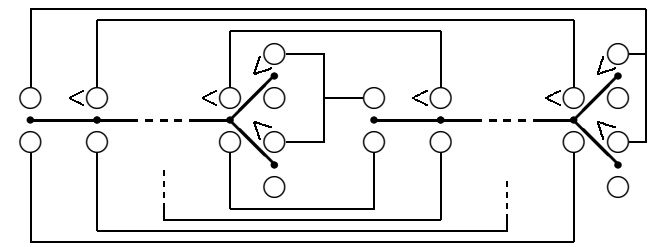

S-10. $\operatorname{ad}^{\mathrm{y}}(p, p+1), p \geq 3$

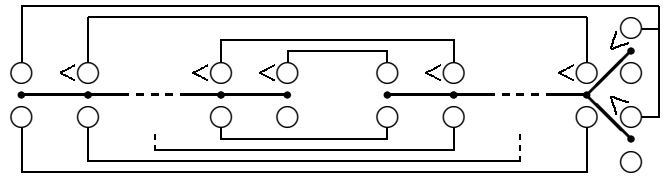


S-11. $\operatorname{ad}^{\mathrm{y}}(p-1, p+1), p \geq 3$

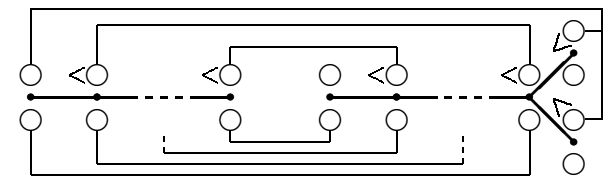

S-12. $\boldsymbol{b d}^{\mathrm{y}}(p, p+1), p \geq 3$

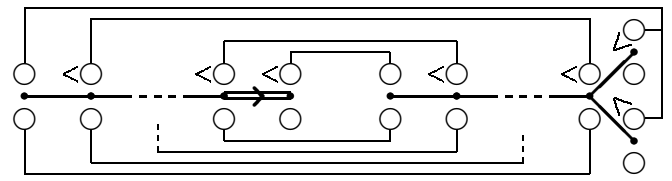

S-13. $\operatorname{ag}^{\mathrm{y}}(2,2)$

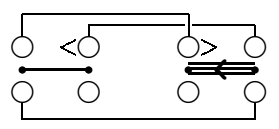

S-14. $\operatorname{ag}^{\mathrm{y}}(1,2)$

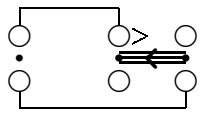

S-15. $\operatorname{bg}^{\mathrm{y}}(2,2)$

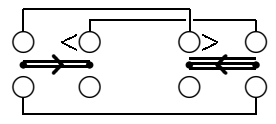

S-16. $\mathrm{g}^{\mathrm{y}}(2,2)$

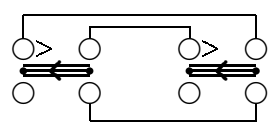

S-17. $\mathbf{a}^{\mathrm{y}}(2 p), p \geq 2$

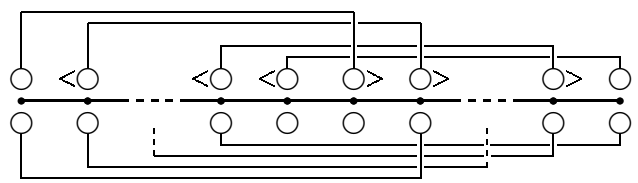

S-18. $b^{\mathrm{y}}(4)$

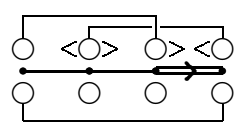

S-19. $c^{y}(4)$

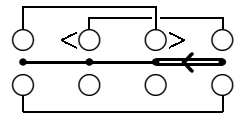

S-20. $d^{\mathrm{y}}(7)$

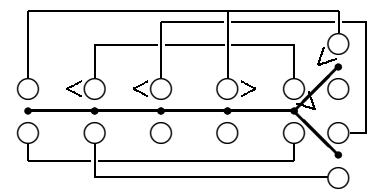


S-21. $\mathrm{f}^{\mathrm{y}}(4)$

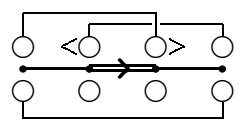

Family z

S-22. $a^{\mathrm{z}}(3,3)$

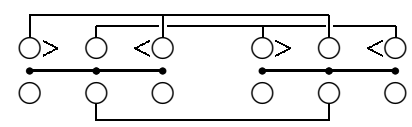

S-23. $a^{\mathrm{z}}(2,3)$

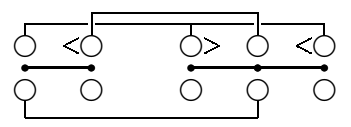

S-24. $a^{z}(1,3)$

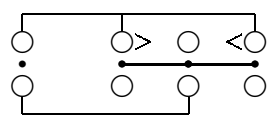

S-25. $a b^{\mathrm{z}}(3,3)$

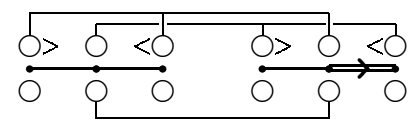

S-26. $a b^{\mathrm{z}}(3,2)$

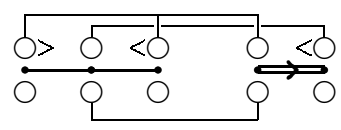

S-27. $a b^{z}(2,3)$

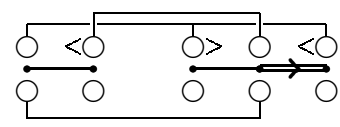

S-28. $a^{\mathrm{z}}(1,3)$

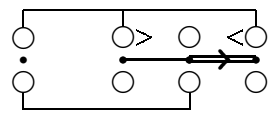

$\mathbf{S}-\mathbf{2 9} \cdot \boldsymbol{b}^{\mathrm{z}}(3,3)$

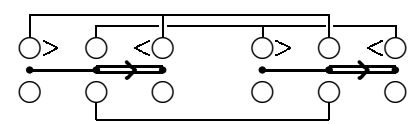

S-30. $b^{z}(2,3)$

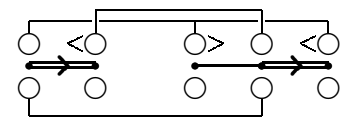

S-31. $\mathrm{ac}^{\mathrm{z}}(1,3)$

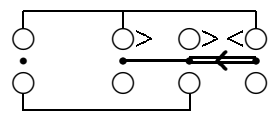


S-32. $d^{\mathrm{z}}(4,4)$

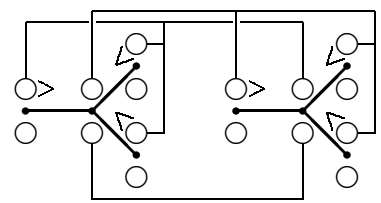

S-33. $\operatorname{ad}^{\mathrm{z}}(3,4)$

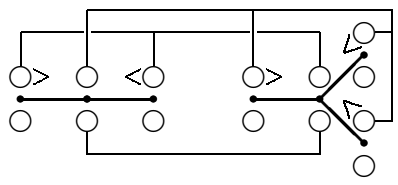

S-34. $\operatorname{ad}^{\mathrm{z}}(2,4)$

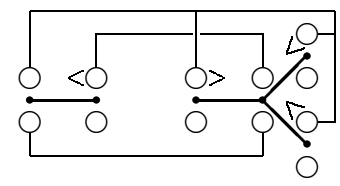

S-35. $\operatorname{ad}^{\mathrm{z}}(1,4)$

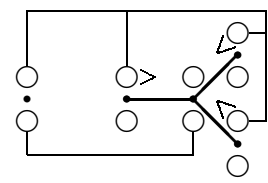

S-36. $b^{z}(3,4)$

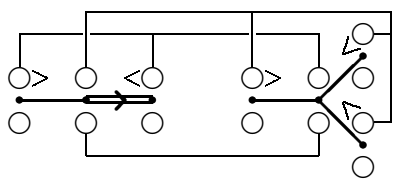

S-37. $b d^{z}(2,4)$

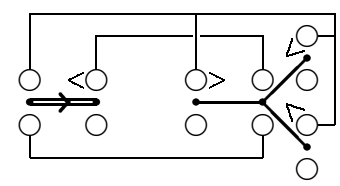

S-38. $\operatorname{ag}^{\mathbf{z}}(3,2)$

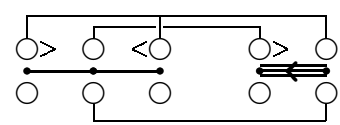

S-39. $\operatorname{bg}^{\mathrm{z}}(3,2)$

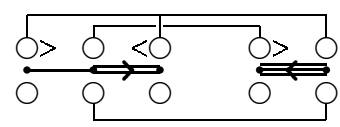

S-40. $\operatorname{dg}^{\mathrm{z}}(4,2)$

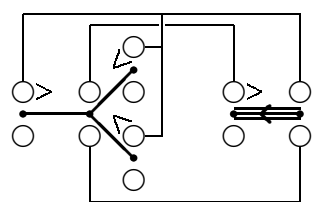


S-41. $a^{z}(6)$

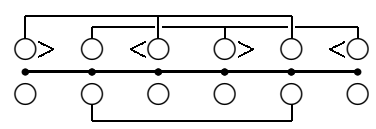

S-42. $\mathrm{a}^{\mathrm{z}}(5)$

$$
\begin{array}{cccccc}
\hline>>0 & 0 & 0 & 0> & 0 \\
\hline 0 & 0 & 0 & 0 & 0
\end{array}
$$

S-43. $b^{z}(6)$

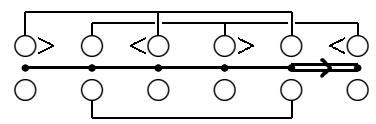

S-44. $b^{z}(5)$

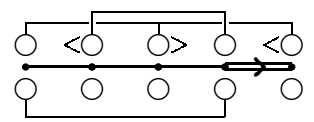

S $-45 \cdot c^{\mathrm{z}}(5)$

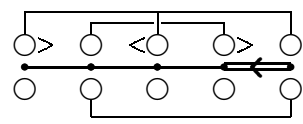

S-46. $d^{z}(7)$

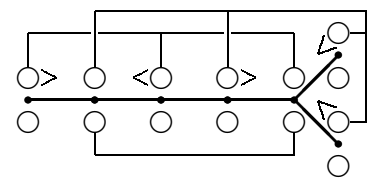

$\mathrm{S}-\mathbf{4 7} \cdot \mathrm{d}^{\mathrm{z}}(6)$

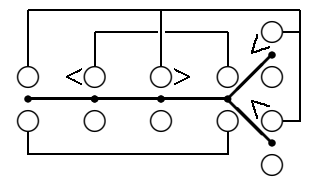

Family u

S-48. $a^{\mathrm{u}}(7)$

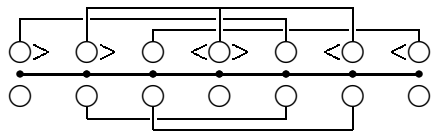

S-49. $a^{\mathrm{u}}(6)$

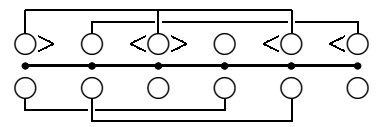

S-50. $\mathrm{a}^{\mathrm{u}}(5)$

$$
\begin{array}{rrrrr}
0 & 0 & 0 & 0 & 0 \\
0>0 & 0 & 0 & 0
\end{array}
$$


S-51. $b^{\mathrm{u}}(7)$

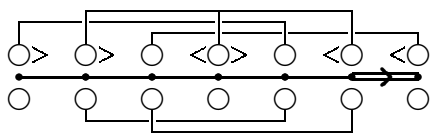

S-52. $b^{\mathrm{u}}(6)$

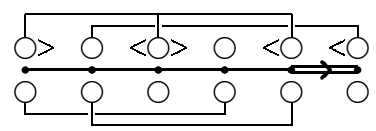

S-53. $\mathrm{c}^{\mathrm{u}}(5)$

$$
\begin{array}{rrr}
0>0 & 0 & 0 \\
0 & 0 & 0
\end{array}
$$

S-54. $d^{\mathrm{u}}(8)$

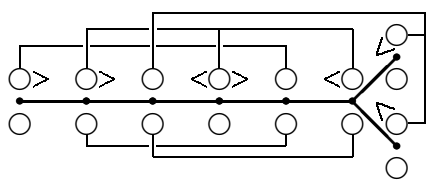

S-55. $\mathrm{d}^{\mathrm{u}}(7)$

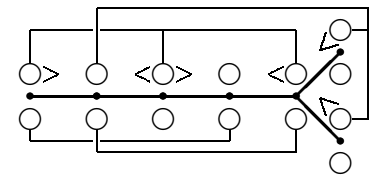

Family v

S-56. $d^{v}(8)$

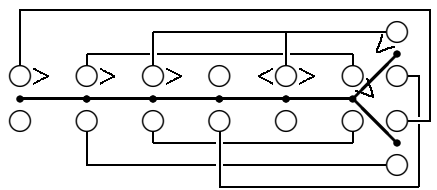

S-57. $e^{v}(8)$

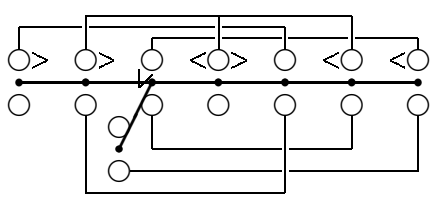

S-58. $d^{\mathrm{v}}(7)$

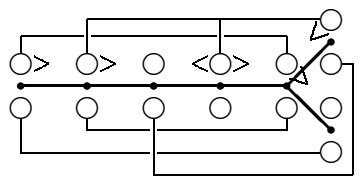

Family w

S-59. $b^{\mathrm{w}}(4)$

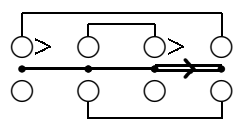


S-60. $\mathrm{f}^{\mathrm{w}}(4)$

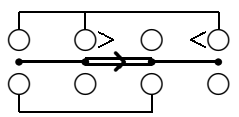

S-61. $b^{\mathrm{w}}(3)$

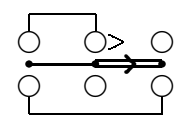

Family p

S-62.

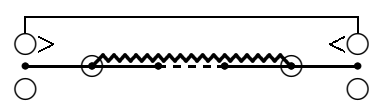

S-63.

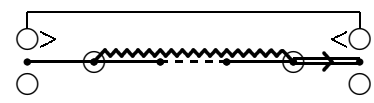

S-64.

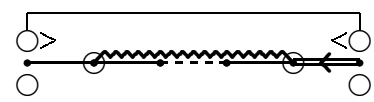

S-65.

$$
\stackrel{0>0}{0<0}
$$

S-66.

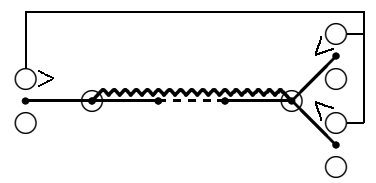

S-67.

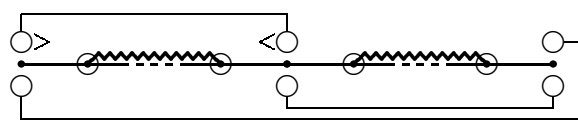

S-68.

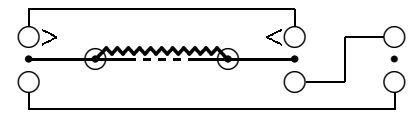

S-69.

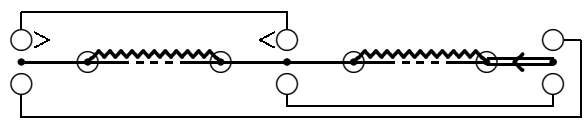

S-70.

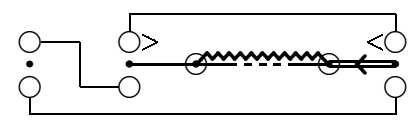

S-71.

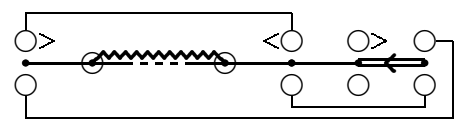


S-72.

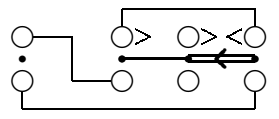

S-73.

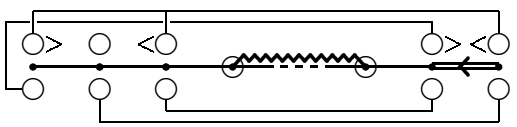

S-74.

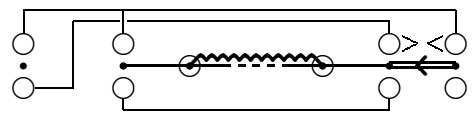

S-75.

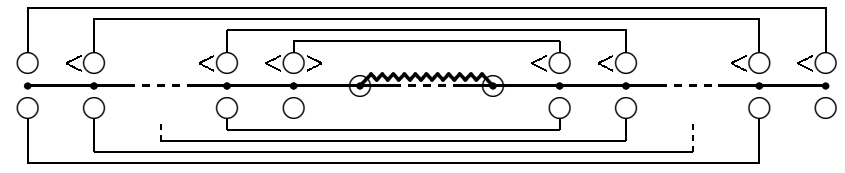

S-76.

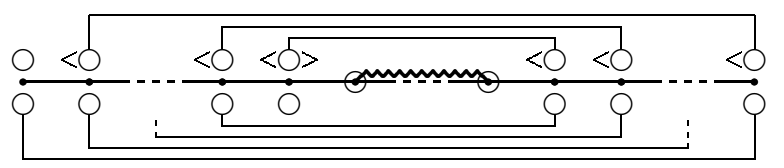

S-77.

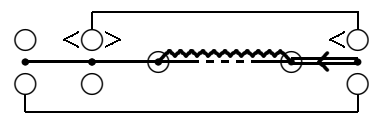

S-78.

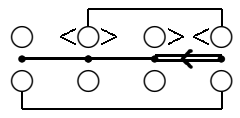

S-79.

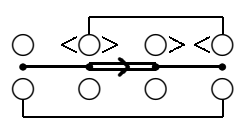

S-80.

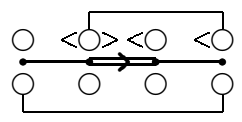

S-81.

$$
\stackrel{0>0<0>0}{\longrightarrow 000}
$$

S-82.

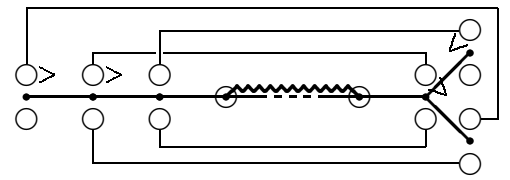


S-83.

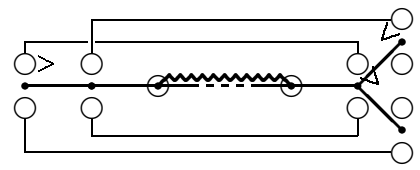

S-84.

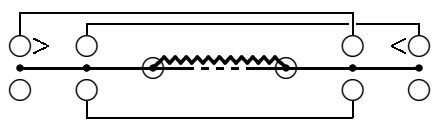

S-85.

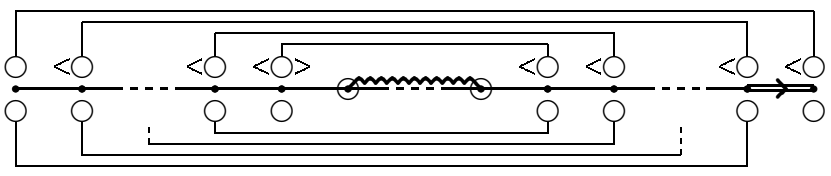

S-86.

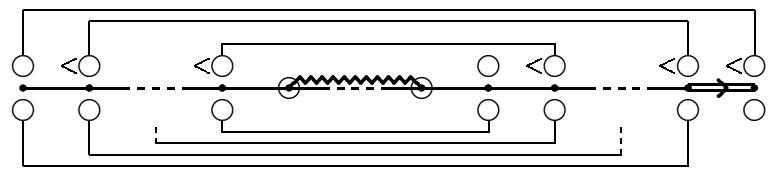

S-87.

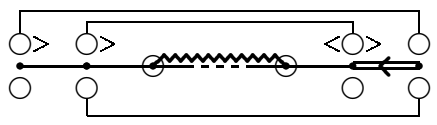

S-88.

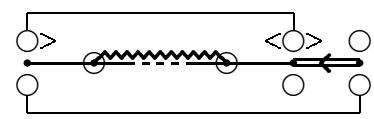

S-89.

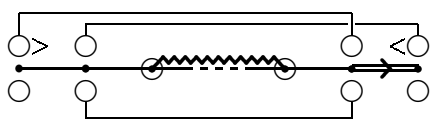

S-90.

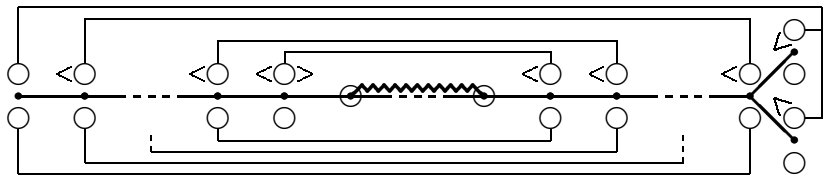

S-91.

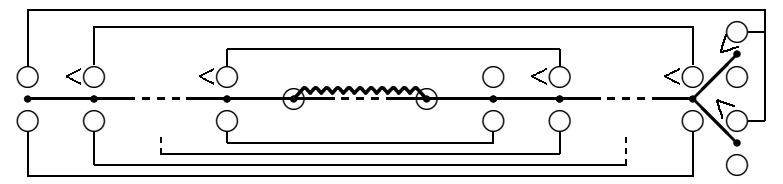

S-92.

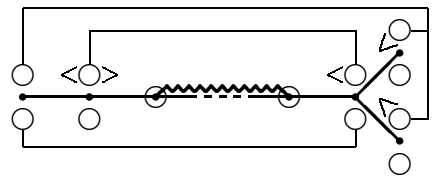


S-93.

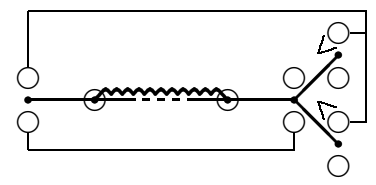

S-94.

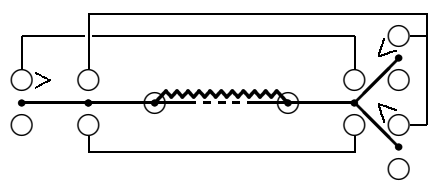

S-95.

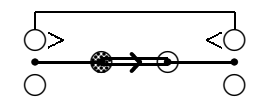

S-96.

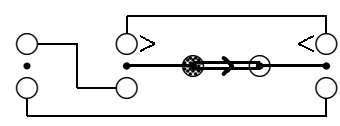

S-97.

$$
\underset{0 \quad 0<0}{\longrightarrow 0}
$$

S-98.

$$
\underset{0>0<0<0}{\longrightarrow}
$$

S-99.

$$
\text { ○> }
$$

S-100.

$$
\begin{array}{lll}
0>0<0<0 \\
\hdashline \underset{0}{0}
\end{array}
$$

S-101.

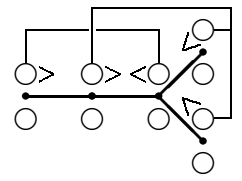

S-102.

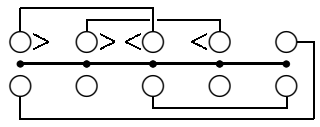

S-103.

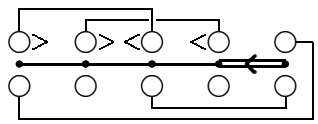


S-104.

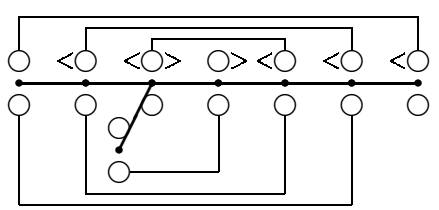

S-105.

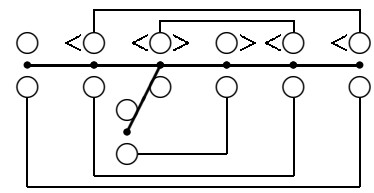

S-106.

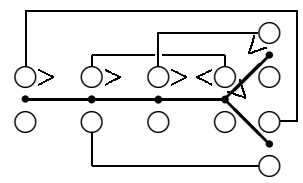

S-107.

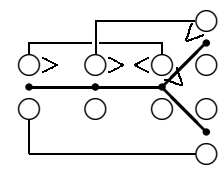

Family q

S-108.

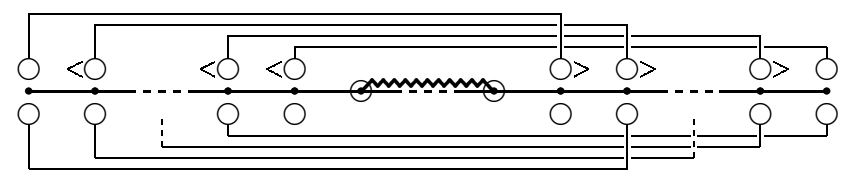

S-109.

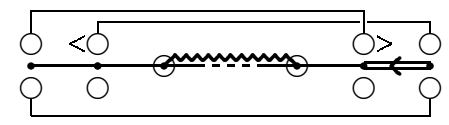

S-110.

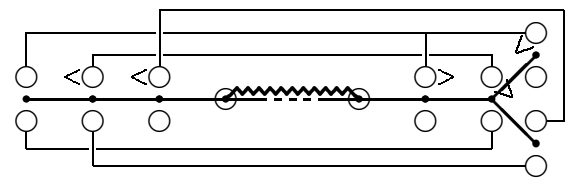

S-111.

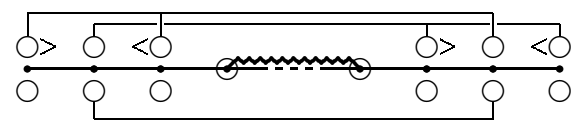

S-112.

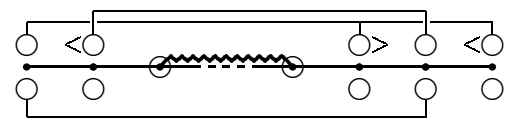

S-113.

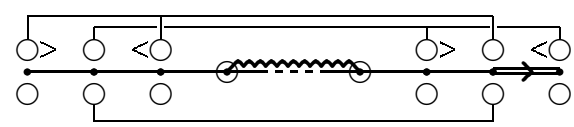


S-114.

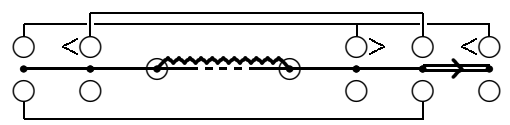

S-115.

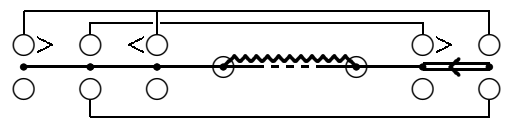

S-116.

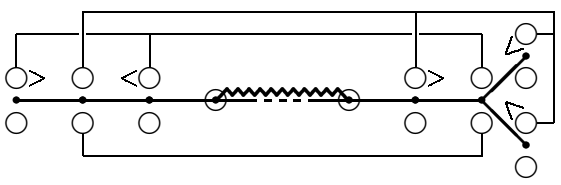

S-117.

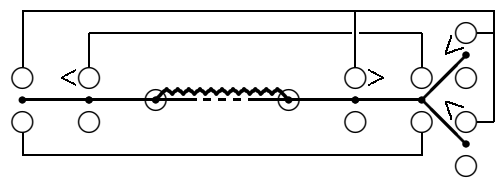

S-118.

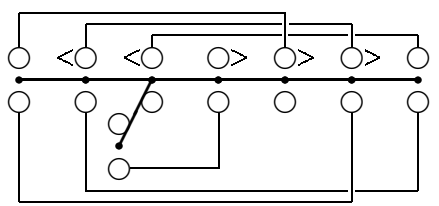

S-119.

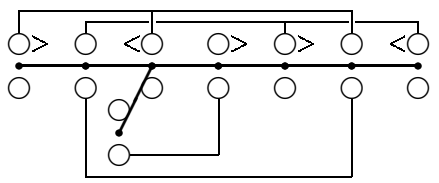

S-120.

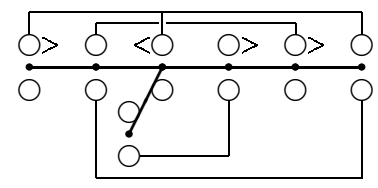

S-121.

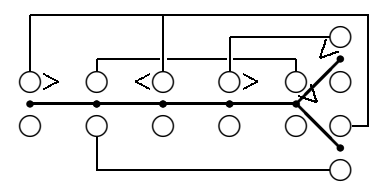

S-122.

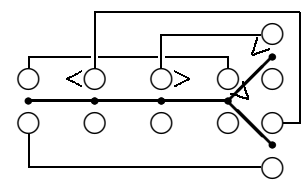


Clan T.

T-1.

$\mathrm{a}^{\mathrm{m}}(n)$

$\mathrm{b}^{\mathrm{m}}(n)$

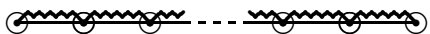

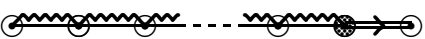

$\mathrm{c}^{\mathrm{m}}(n)$

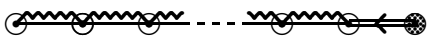

$\mathrm{d}^{\mathrm{m}}(n)$

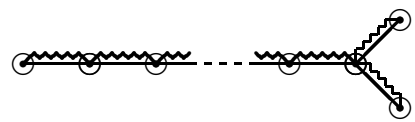

$\mathrm{e}^{\mathrm{m}}(n)$

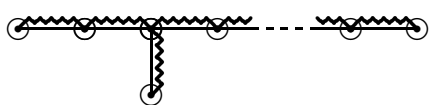

$\mathrm{f}^{\mathrm{m}}(4)$

$\mathrm{sman}$

$g^{m}$

$\rightleftharpoons$

Family a

$\mathbf{T}-2$.

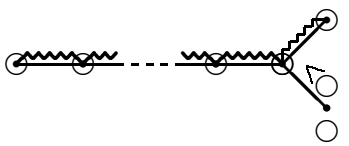

$\mathbf{T}-\mathbf{3}$.

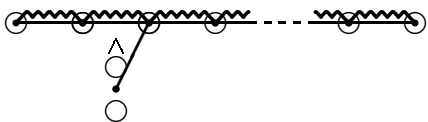

$\mathrm{T}-4$.

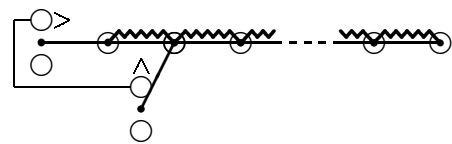

$\mathbf{T}-\mathbf{5}$.

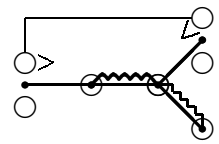

T-6.

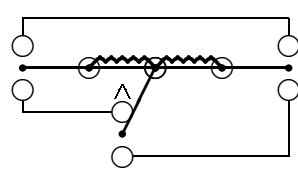


$\mathbf{T}-\mathbf{7}$.

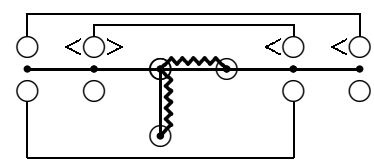

$\mathbf{T}-\mathbf{8}$.

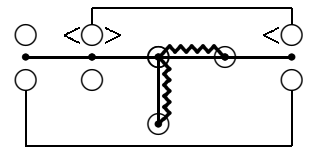

$\mathbf{T}-\mathbf{9}$.

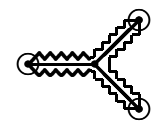

$\mathbf{T}-\mathbf{1 0}$.

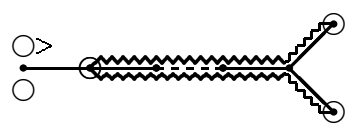

$\mathrm{T}-11$

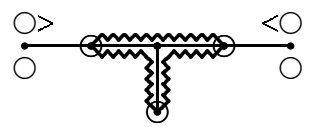

$\mathbf{T}-12$.

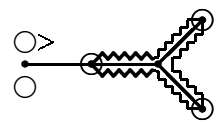

$\mathrm{T}-13$.

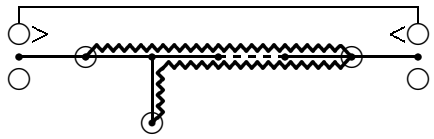

$\mathrm{T}-14$

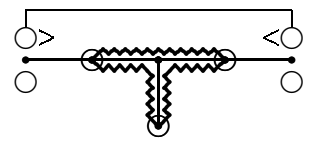

Family b

$\mathbf{T}-15$.

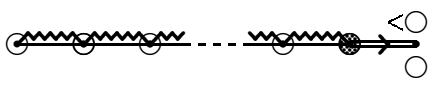

$\mathbf{T}-\mathbf{1 5}^{\prime}$

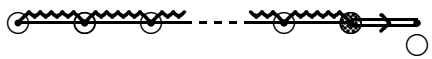

$\mathrm{T}-16$.

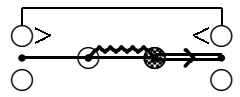

$\mathbf{T}-17$.

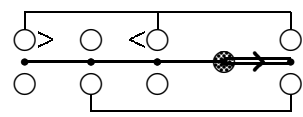


$\mathrm{T}-18$.

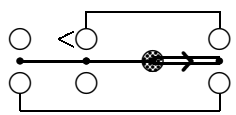

$\mathrm{T}-19$.

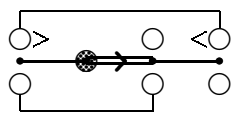

T-20.

$$
\underset{0}{<0}<0
$$

$\mathbf{T}-21$.

$$
\underset{0}{\longrightarrow 0} \underset{0}{\longrightarrow}
$$

Family c

T-22.

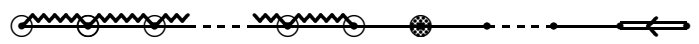

T-23.

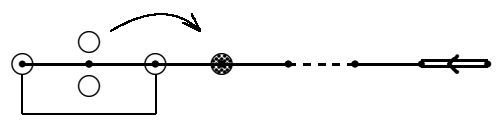

T-24.

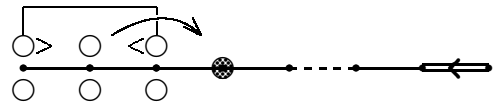

T-25.

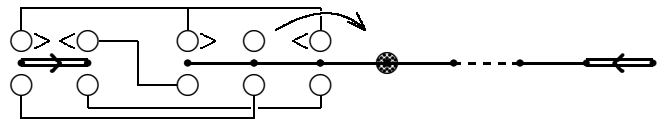

T-26.

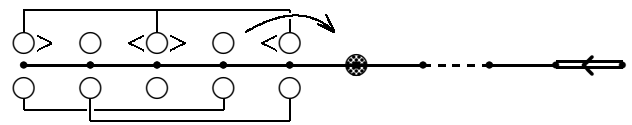

T-27.

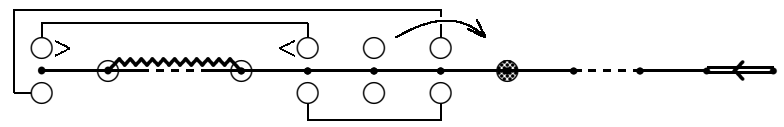

T-28.

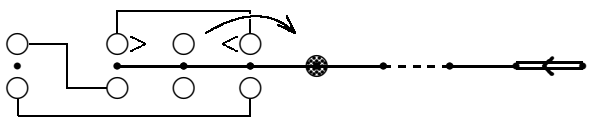

T-29.

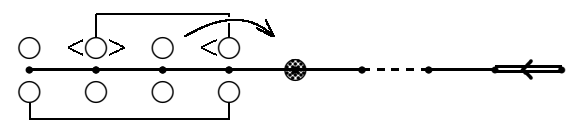


2.2. Primitive positive 1-combs. Here we introduce the notion of a primitive positive 1-comb of a spherical $R$-system and give the list of all the spherical $R$ systems with a primitive positive 1-comb, for any root system $R$.

Definition 2.11 (Primitive positive 1-comb). Let $\mathscr{S}=\left(S^{p}, \Sigma, A\right)$ be a spherical $R$-system. A positive 1-comb $D \in A$ of $\mathscr{S}$ is called primitive if $\mathscr{S}$ is cuspidal, not decomposable and without tails.

Notice that any spherical system admits at most one primitive positive 1-comb.

Theorem 2.12. The families $p$ and $q$, given below, contain all the spherical $R$ systems of rank $>2$ with a primitive positive 1-comb, up to external automorphism of $R$.

The proof is in Section 2.5

\section{Family p}

ppc-1.

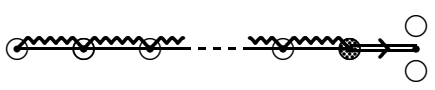

ppc-2.

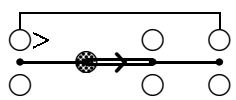

ppc-3.

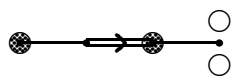

ppc-4.

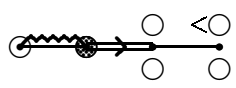

\section{Family q}

ppc-5.

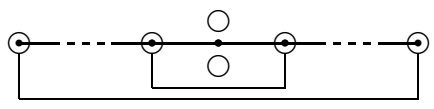

ppc-6.

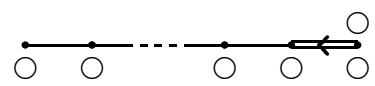

ppc-7.

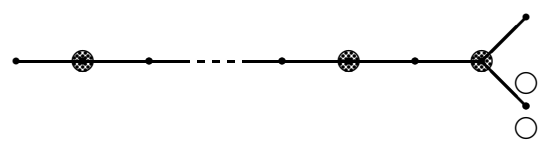

ppc-8.

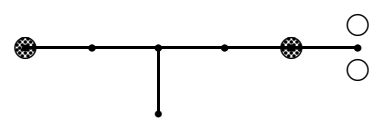

ppc-9.

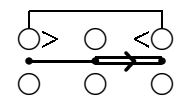


ppc-10.

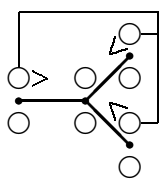

ppc-11.

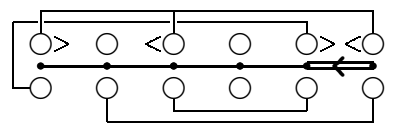

ppc-12.

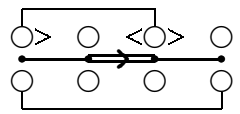

ppc-13.

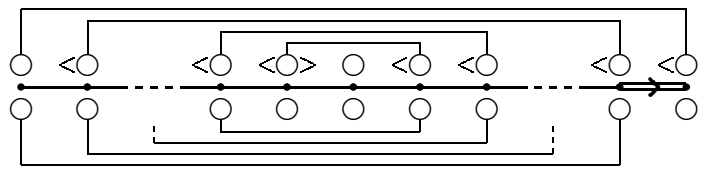

ppc-14.

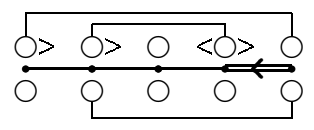

ppc-15.

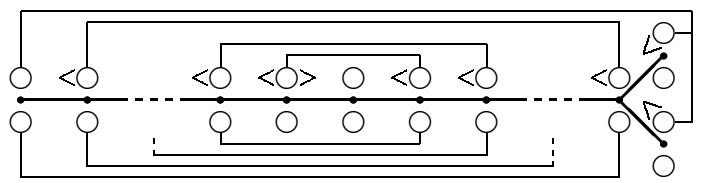

ppc-16.

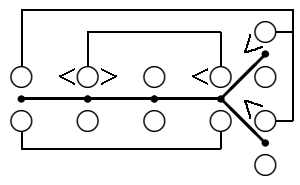

2.3. Minimal quotients. The minimal quotients (see Section 1.3) of all the primitive spherical systems and of all the spherical systems with a primitive positive 1-comb (of rank > 2) can be explicitly computed. In particular, the following can be checked case-by-case.

Remark 2.13. All the distinguished subsets of colors of the primitive spherical systems or of the spherical systems with a primitive positive 1-comb are good.

Here are some complementary remarks on the structure of some classes of primitive spherical systems with special attention to their quotients.

First, we remark that all the primitive spherical systems have defect $\leq 2$, as can be checked on the list. 
Clan $R$. The members of the clan $\mathrm{R}$ have defect 0 or 1 . Those of defect 1 are exactly the cases R-2, R-6, R-11, R-12 and R-13.

All the members $\mathscr{S}$ of defect 0 have a unique minimal distinguished subset of colors, the full set of colors $\Delta$. Such a distinguished subset of colors is homogeneous and $\mathscr{S} / \Delta=(S, \emptyset, \emptyset)$.

All the members $\mathscr{S}$ of defect 1 have two colors $D_{1}, D_{2}$ such that $c\left(D_{1}, \sigma\right)=$ $c\left(D_{2}, \sigma\right)$ for all spherical roots $\sigma$, and have exactly two minimal distinguished subsets of colors, namely $\Delta \backslash\left\{D_{1}\right\}$ and $\Delta \backslash\left\{D_{2}\right\}$. Such subsets of colors are homogeneous and such that $S^{p} /\left(\Delta \backslash\left\{D_{i}\right\}\right)=\{\alpha\}$ with $D_{i} \in \Delta(\alpha)$.

Clan S. All the members of the family $x$ have defect 0 . All the members of the families y, z, u, v, w have defect 1 .

Clan T. All the members of the clan $\mathrm{T}$ with only spherical roots of type $a(\mathrm{~m})$ have the same rank, the same number of colors and the same Cartan pairing of some corresponding members of the clan S; therefore such spherical systems have corresponding minimal quotients also.

Let us now consider the members of the family c. The case $\mathrm{T}-22$ is a generalization of $c^{\mathrm{m}}(n)$, with similar quotients. Let us restrict our attention to the remaining cases.

The case T-23 below (on the right-hand side), after "collapsing" the spherical root of type $c(m)$, corresponds to the primitive spherical system below (on the left-hand side); the minimal quotients of the former are in correspondence with the minimal quotients of the latter:
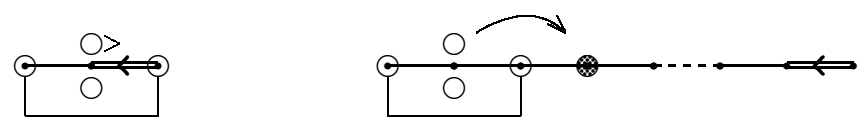

The same relation holds for the other members of the family $\mathrm{c}$ and the following primitive spherical systems, respectively: S-65] S-3 namely $b c^{\times}(2,3)$, S-53 namely $c^{\mathrm{u}}(5), \mathrm{S}-71, \mathrm{~S}-72$ and S-78.

2.4. General spherical systems. A clear consequence of Theorems 2.10 and 2.12 is that a general spherical system can be obtained from primitive spherical systems, spherical systems with a primitive positive 1-comb and "trivial" spherical systems (i.e. rank 0 spherical $R$-systems with $S^{p}=S$ ) by applying recursively four procedures, which we illustrate in the following: parabolic induction, composition, join of positive 1-combs and addition of tails.

Parabolic induction. Let $S^{\prime}$ be a subset of $S$ and $R^{\prime}$ the corresponding root subsystem of $R$.

Let $\mathscr{S}^{\prime}=\left(\left(S^{\prime}\right)^{p}, \Sigma^{\prime}, A^{\prime}\right)$ be a spherical $R^{\prime}$-system. The triple $\left(\left(S^{\prime}\right)^{p}, \Sigma^{\prime}, A^{\prime}\right)$ can also be considered as a spherical $R$-system; it is called the spherical $R$-system obtained from $\mathscr{S}^{\prime}$ by parabolic induction.

Once having recalled Definition 1.7 it is obvious that the parabolic induction is the inverse procedure of the localization in the following sense:

(1) Let $\mathscr{S}^{\prime}$ be a spherical $R^{\prime}$-system, and let $\mathscr{S}$ be the spherical $R$-system obtained from $\mathscr{S}^{\prime}$ by parabolic induction. Then $\mathscr{S}^{\prime}$ equals the spherical $R^{\prime}$-system obtained from $\mathscr{S}$ by localization. 
(2) Let $\mathscr{S}=\left(S^{p}, \Sigma, A\right)$ be a spherical $R$-system, and let $\mathscr{S}^{\prime}$ be the spherical $R^{\prime}$-system obtained from $\mathscr{S}$ by localization. Then $\mathscr{S}$ equals the spherical $R$-system obtained from $\mathscr{S}^{\prime}$ by parabolic induction if

$$
S^{p} \cup \bigcup_{\sigma \in \Sigma} \operatorname{supp} \sigma \subset S^{\prime}
$$

Composition. Recall Definition 2.1. Let $\mathscr{S}=\left(S^{p}, \Sigma, A\right)$ be a spherical $R$-system. Let $\Delta^{\prime}$ and $\Delta^{\prime \prime}$ be good distinguished subsets of colors that decompose $\mathscr{S}$. Then:

- $\left(\left(S^{p} / \Delta^{\prime} \cup \Delta^{\prime \prime}\right) \backslash\left(S^{p} / \Delta^{\prime}\right)\right) \perp\left(\left(S^{p} / \Delta^{\prime} \cup \Delta^{\prime \prime}\right) \backslash\left(S^{p} / \Delta^{\prime \prime}\right)\right)$ and

- $\left(\left(\Sigma / \Delta^{\prime}\right) \backslash\left(\Sigma / \Delta^{\prime} \cup \Delta^{\prime \prime}\right)\right) \cap\left(\left(\Sigma / \Delta^{\prime \prime}\right) \backslash\left(\Sigma / \Delta^{\prime} \cup \Delta^{\prime \prime}\right)\right)=\emptyset$.

Proposition 2.14. Let $\mathscr{S}_{1}=\left(S_{1}^{p}, \Sigma_{1}, A_{1}\right)$ and $\mathscr{S}_{2}=\left(S_{2}^{p}, \Sigma_{2}, A_{2}\right)$ be spherical $R$ systems with set of colors $\Delta_{1}$ and $\Delta_{2}$, respectively. Let $\Delta^{\prime \prime} \subset \Delta_{1}$ and $\Delta^{\prime} \subset \Delta_{2}$ be good distinguished subsets with $\mathscr{S}_{1} / \Delta^{\prime \prime}=\mathscr{S}_{2} / \Delta^{\prime}$, say equal to $\left(S_{1,2}^{p}, \Sigma_{1,2}, A_{1,2}\right)$. Assume that

- $\left(S_{1,2}^{p} \backslash S_{1}^{p}\right) \perp\left(S_{1,2}^{p} \backslash S_{2}^{p}\right)$ and

- $\left(\Sigma_{1} \backslash \Sigma_{1,2}\right) \cap\left(\Sigma_{2} \backslash \Sigma_{1,2}\right)=\emptyset$.

Set

- $S^{p}=S_{1}^{p} \cap S_{2}^{p}$,

- $\Sigma=\left(\Sigma_{1} \backslash \Sigma_{1,2}\right) \cup\left(\Sigma_{2} \backslash \Sigma_{1,2}\right) \cup\left(\Sigma_{1} \cap \Sigma_{2}\right)$,

- $A=A_{1} \cup A_{2}$ (identifying $A_{1} / \Delta^{\prime \prime} \subset A_{1}$ with $A_{2} / \Delta^{\prime} \subset A_{2}$ ).

Then

- $\mathscr{S}=\left(S^{p}, \Sigma, A\right)$ is a spherical $R$-system, with set of colors $\Delta=\Delta_{1} \cup \Delta_{2}$ (identifying $\Delta_{1} \backslash \Delta^{\prime \prime}$ with $\Delta_{2} \backslash \Delta^{\prime}$ ),

- $\Delta^{\prime}$ and $\Delta^{\prime \prime}$ decompose $\mathscr{S}$,

- $\mathscr{S} / \Delta^{\prime}=\mathscr{S}_{1}$ and $\mathscr{S} / \Delta^{\prime \prime}=\mathscr{S}_{2}$.

Proof. Let us consider $\mathscr{S}_{2}$ and its quotient $\mathscr{S}_{1,2}=\mathscr{S}_{2} / \Delta^{\prime}$, with set of spherical roots $\Sigma_{1,2}=\Sigma_{2} / \Delta^{\prime}$. If $D \in \Delta^{\prime} \subset \Delta_{2}$, then $c_{2}(D, \sigma)=0$ for all $\sigma \in \Sigma_{2} \cap \Sigma_{1,2}$. In particular, we are allowed to set $c(D, \sigma)=0$ for all $\sigma \in \Sigma_{1} \backslash \Sigma_{1,2}$. On the other hand, for all $\alpha \in S \cap \Sigma_{2} \backslash \Sigma_{1,2}$ one has $A_{2}(\alpha) \cap \Delta^{\prime} \neq \emptyset$. By considering $\mathscr{S}_{1}$ and its quotient $\mathscr{S}_{1,2}=\mathscr{S}_{1} / \Delta^{\prime \prime}$, one gets the two analogous symmetric assertions.

This shows that the restricted Cartan pairing of $\mathscr{S}$ is well defined. Indeed, let $D$ be in $A(\alpha)$. If $\alpha \in \Sigma_{1} \cap \Sigma_{2} \subset \Sigma_{1,2}$, then $A(\alpha)$ can be identified with $A_{1}(\alpha)=A_{2}(\alpha)=A_{1,2}(\alpha)$; thus set $c(D, \sigma)=c_{i}(D, \sigma)$ if $\sigma \in \Sigma_{i}, i=1,2$, and notice that $c_{1}(D, \sigma)=c_{2}(D, \sigma)$ if $\sigma \in \Sigma_{1} \cap \Sigma_{2} \subset \Sigma_{1,2}$. If $\alpha \in \Sigma_{2} \backslash \Sigma_{1,2}$, then, for all $\sigma \in \Sigma_{1} \backslash \Sigma_{1,2}, c(D, \sigma)=0$ if $D \in \Delta^{\prime}$ or $c(D, \sigma)=\left\langle\alpha^{\vee}, \sigma\right\rangle$ if $D \notin \Delta^{\prime}$ (notice that if $A_{2}(\alpha) \subset \Delta^{\prime}$, then $\alpha \perp \sigma$ for all $\left.\sigma \in \Sigma_{1} \backslash \Sigma_{1,2}\right)$; finally, $c(D, \sigma)=c_{2}(D, \sigma)$ for all $\sigma \in \Sigma \cap \Sigma_{2}$.

Analogously, the axioms of the spherical system for $\mathscr{S}$ and the rest of the statement follow.

Under the hypotheses of the above proposition we say that the spherical system $\mathscr{S}$ is obtained by composing the spherical systems $\mathscr{S}_{1}$ and $\mathscr{S}_{2}$.

The converse is immediate: if $\mathscr{S}$ is a spherical $R$-system and $\Delta^{\prime}$ and $\Delta^{\prime \prime}$ are good distinguished subsets of colors that decompose $\mathscr{S}$, then the spherical $R$-system obtained by composing $\mathscr{S} / \Delta^{\prime}$ and $\mathscr{S} / \Delta^{\prime \prime}$ is equal to $\mathscr{S}$. 
Join of positive 1-combs. Recall Definition 1.9. Let $\mathscr{S}=\left(S^{p}, \Sigma, A\right)$ be a spherical $R$-system with $D_{1}, \ldots, D_{m}$ positive combs with disjoint sets $S_{D_{1}}, \ldots, S_{D_{m}}$. Let $S_{1}, \ldots, S_{k}$ be disjoint subsets of $S$ such that $S_{1} \cup \ldots \cup S_{k}=S_{D_{1}} \cup \ldots \cup S_{D_{m}}$. The spherical $R$-system $\left(S^{p}, \Sigma, A^{\prime}\right)$, where $A^{\prime}$ is obtained from $A$ by replacing the positive combs $D_{1}, \ldots, D_{m}$ by other positive combs, say $D_{1}^{\prime}, \ldots, D_{k}^{\prime}$, with $S_{D_{i}^{\prime}}=S_{i}$, $1 \leq i \leq k$, is well defined. Moreover, this procedure is clearly reversible.

In particular, if $\alpha_{1}, \ldots, \alpha_{m}$ are pairwise distinct simple roots and $D_{\alpha_{1}}^{+}, \ldots, D_{\alpha_{m}}^{+}$ are positive 1-combs, it is possible to join these positive 1-combs: namely replace them by a unique positive $m$-comb $D^{\prime}$ with $S_{D^{\prime}}=\left\{\alpha_{1}, \ldots, \alpha_{m}\right\}$.

Addition of tails.

Definition 2.15. A color $D$ is called free if there exists at most one spherical root $\sigma$ with $c(D, \sigma)>0$.

Recall Definition 2.5 and its notation.

Proposition 2.16. Let $\mathscr{S}$ be a spherical $R$-system with a tail $\sigma \in \Sigma$.

(1) If $\sigma$ is of type $b(m), 2 b(m)$ or $d(m)$, then there exist at most one spherical root $\sigma^{\prime}$ with $\alpha_{n-m} \in \operatorname{supp} \sigma^{\prime}$ and one free color $D^{\prime}$ such that $c\left(D^{\prime}, \sigma\right)<0$ and $c\left(D^{\prime}, \sigma^{\prime}\right)=1$. The spherical root $\sigma^{\prime}$ is of type $a(k), k \geq 1$.

(2) If $\sigma$ is of type $c(m)$, then there exist at most one spherical root $\sigma^{(0)}$ with $\alpha_{n-m+1} \in \operatorname{supp} \sigma^{(0)}$, one spherical root $\sigma^{\prime}$ with $\alpha_{n-m} \in \operatorname{supp} \sigma^{\prime}$ and one free color $D^{\prime}$ such that $c\left(D^{\prime}, \sigma\right)<0$ and $c\left(D^{\prime}, \sigma^{\prime}\right)=1$. The spherical root $\sigma^{(0)}$ can be either of type a(1), of type aa, of type $2 a$ or of type $a(2)$. More precisely, if $\sigma^{(0)}$ is of type $a(2)$, then $\sigma^{(0)}=\sigma^{\prime}$; if $\sigma^{(0)}$ is of type $2 a$, there exists no $\sigma^{\prime}$ as above; otherwise, if $\sigma^{(0)}$ is of type aa or a(1), then $\alpha_{m-n+1} \notin \operatorname{supp} \sigma^{\prime}$ and $\sigma^{\prime}$ is of type $a(k)$ with $k \geq 1$.

Proof. The less evident case is that of type $b(1)$, with $\sigma^{\prime}$ of type $a(1)$ : let us restrict ourselves to this. If there exists a simple spherical root $\sigma^{\prime \prime} \neq \sigma^{\prime}$ with $c\left(D^{\prime}, \sigma^{\prime \prime}\right)=1$, then there exists a color $D \in A(\sigma) \cap A\left(\sigma^{\prime \prime}\right)$; hence $\sigma$ cannot be a tail.

Vice versa, if a spherical $R^{\prime}$-system satisfies the conditions obtained in the above proposition it is possible to add a tail of type $b(m), 2 b(m)$ or $d(m)$, in the following sense.

Let $\mathscr{S}^{\prime}=\left(\left(S^{\prime}\right)^{p}, \Sigma^{\prime}, A^{\prime}\right)$ be a spherical $R^{\prime}$-system. Let $\alpha_{1}, \ldots, \alpha_{l}$ be the simple roots of an irreducible component of type A of $R^{\prime}$. Assume that there exists a spherical root $\sigma^{\prime} \in \Sigma^{\prime}$ with $\alpha_{l} \in \operatorname{supp} \sigma^{\prime}$. Furthermore, suppose: (i) $\sigma^{\prime}$ is of type $a(k), k \geq 1$, and (ii) there exists a free color $D^{\prime} \in \Delta\left(\alpha_{l}\right)$ with $c\left(D^{\prime}, \sigma^{\prime}\right)=1$ ((ii) follows from (i) if $k>1$ ). Then it is possible to add a tail $\sigma$ of type $b(m), 2 b(m)$ or $d(m)$ to $\mathscr{S}^{\prime}, \operatorname{supp} \sigma=\left\{\alpha_{l+1}, \ldots, \alpha_{l+m}\right\}$. Indeed, the spherical $R$-system $\mathscr{S}$ with $\Sigma=\Sigma^{\prime} \cup\{\sigma\}$ and $D^{\prime}$ a unique color such that $c\left(D^{\prime}, \sigma\right)<0$ is well defined and $\sigma$ is actually a tail. The fact that $\sigma$ is a tail, i.e. that there exists a good distinguished subset of colors $\Delta^{\prime}$ as in Definition 2.5] can be checked (see Lemma 2.22) on the primitive spherical systems.

The case of the tail of type $c(m)$ is slightly more complicated.

Let $\mathscr{S}^{\prime}=\left(\left(S^{\prime}\right)^{p}, \Sigma^{\prime}, A^{\prime}\right)$ be a spherical $R^{\prime}$-system. Let $\alpha_{1}, \ldots, \alpha_{l+1}$ be the simple roots of an irreducible component of type $\mathrm{A}$ of $R^{\prime}$. Assume that there exist a spherical root $\sigma^{(0)} \in \Sigma^{\prime}$ with $\alpha_{l+1} \in \operatorname{supp} \sigma^{(0)}$ and a spherical root $\sigma^{\prime} \in \Sigma^{\prime}$ with $\alpha_{l} \in \operatorname{supp} \sigma^{\prime}$. Furthermore, suppose: (i) $\sigma^{\prime}$ is of type $a(k), k \geq 1$, and (ii) 
there exists a free color $D^{\prime} \in \Delta\left(\alpha_{l}\right)$ with $c\left(D^{\prime}, \sigma^{\prime}\right)=1$. Then it is possible to add a spherical root $\sigma$ of type $c(m)$ to $\mathscr{S}^{\prime}, \operatorname{supp} \sigma=\left\{\alpha_{l+1}, \ldots, \alpha_{l+m}\right\}$. Indeed, the spherical $R$-system $\mathscr{S}$ with $\Sigma=\Sigma^{\prime} \cup\{\sigma\}$ and $D^{\prime}$ a unique color such that $c\left(D^{\prime}, \sigma\right)<0$ is well defined. The spherical root $\sigma$ is not necessarily a tail; i.e. a good distinguished subset of colors $\Delta^{\prime}$ as in Definition 2.5 does not necessarily exist. If $l=0$, there exists neither $\sigma^{\prime}$ nor $D^{\prime}$ but the spherical $R$-system with a spherical root $\sigma$ of type $c(m)$ as above is still well defined and $\sigma$ is always a tail.

Now recall Definition 2.7. The behavior of tails of exceptional type is similar to that of tails of type $2 b(m)$ or $d(m)$. The following is therefore given without proof.

Proposition 2.17. Let $\mathscr{S}$ be a spherical R-system with a tail $\widetilde{\Sigma} \subset \Sigma$ of type $(a a, a a),(d 3, d 3),(d 5, d 5)$ or $(2 a, 2 a)$. Then there exist at most one spherical root $\sigma^{\prime}$ not orthogonal to $\widetilde{\Sigma}$ and one free color $D^{\prime}$ such that $c\left(D^{\prime}, \sigma_{i}\right)<0$ for some $i=1,2$ and $c\left(D^{\prime}, \sigma^{\prime}\right)=1$. The spherical root $\sigma^{\prime}$ is of type $a(k), 1 \leq k \leq 2$.

Analogously, let $\mathscr{S}^{\prime}=\left(\left(S^{\prime}\right)^{p}, \Sigma^{\prime}, A^{\prime}\right)$ be a spherical $R^{\prime}$-system. Let $\beta_{1}, \beta_{2}$ be the simple roots of an irreducible component of type A of $R^{\prime}$. Assume that there exists a spherical root $\sigma^{\prime} \in \Sigma^{\prime}$ with $\beta_{2} \in \operatorname{supp} \sigma^{\prime}$. Furthermore, suppose: (i) $\sigma^{\prime}$ is of type $a(k), 1 \leq k \leq 2$, and (ii) there exists a free color $D^{\prime} \in \Delta\left(\beta_{2}\right)$ with $c\left(D^{\prime}, \sigma^{\prime}\right)=1$ ((ii) follows from (i) if $k>1)$. Then it is possible to add a tail of type (aa,aa), (d3,d3), $(d 5, d 5)$ or $(2 a, 2 a)$ to $\mathscr{S}^{\prime}$. Indeed, the spherical $R$-system $\mathscr{S}$ with $\Sigma=\Sigma^{\prime} \cup \widetilde{\Sigma}$ and $D^{\prime}$ a unique color such that $c\left(D^{\prime}, \sigma_{i}\right)<0$, for some $i=1,2$, is well defined and $\widetilde{\Sigma}$ is actually a tail.

2.5. Color-adjacent spherical roots and gluing. To prove Theorems 2.10 and 2.12 we introduce several technical combinatorial notions. They help us to understand and describe more explicitly the combinatorial structure of a general spherical system.

Color-connected spherical systems. For simplicity, let us assume for the moment that

$$
\text { if } \Delta(\alpha) \neq \emptyset \text {, then } \alpha \text { lies in the support of (at most) one spherical root. }
$$

Two spherical roots $\sigma_{1}$ and $\sigma_{2}$ are called color-adjacent if they satisfy the following: for all colors $D \in \Delta(\alpha)$ for some $\alpha \in \operatorname{supp} \sigma_{i}, c\left(D, \sigma_{j}\right) \neq 0$, for $i \neq j$. In the appendix one can find all the possible pairs of such color-adjacent spherical roots. Two spherical roots will be called color-connected if they satisfy the above relation extended by transitivity. For short, a spherical system will be called color-connected if its spherical roots are pairwise color-connected.

Lemma 2.18. A cuspidal color-connected spherical system (under the condition (2.1) above) of rank $>2$

- is primitive and is either a member of the clan $R$, or a member of the families $x, y, z, u, v, w$ of the clan $S$,

- or is an n-comb.

Proof. It is enough to proceed recursively: assume we have such a color-connected spherical system; then we check if it can be the localization of another colorconnected spherical system with one more spherical root.

Let us start with a spherical root of type $a a$. It can be color-adjacent to a spherical root of type either $a a, 2 a, a(p)(p \geq 1)$ or $b(2)$. If it is color-adjacent to 
a spherical root of type $a(1)$, then the latter cannot be color-adjacent to another spherical root of the same type $a(1)$ by the axiom (A1). Then we can go on recursively, but no other types of spherical roots can occur and we can find all the cuspidal color-connected spherical systems with a spherical root of type $a a$ : the rank $>2$ cases are members of the clan $R$.

Let us start with a spherical root of type $d(m)$. It can be color-adjacent to a spherical root of type either: $d(3), 2 a, a(1), a(3), c(p), 2 b(2)$ (if $m=3), 2 a, a(1)$, $a(5)$ (if $m=4), d(5), 2 a, a(1)$ (if $m=5$ ) or $2 a, a(1)$ (if $m>5$ ). Again a spherical root of type $a(1)$ here cannot be color-adjacent to another spherical root of the same type $a(1)$. We get a list of rank $>2$ cases which is included in the clan R.

Let us start with a spherical root of type $2 a$. It can be color-adjacent to a spherical root of type $2 a, a(1), 2 b(p)(p \geq 2)$. Roots of type $a a$ and $d(p)$ have already been considered. Similarly, we get a list of rank $>2$ cases which is included in the clan $\mathrm{R}$.

We get no new cases by starting with a spherical root of type $2 b(m)$.

If we start with spherical roots of type different from those considered above and different from $a(1)$ we get only rank 1 or rank 2 cases.

Let us restrict ourselves to the cases with only spherical roots of type $a(1)$. Two such roots $\alpha_{1}, \alpha_{2}$ are color-adjacent if and only if $\Delta\left(\alpha_{1}\right) \cap \Delta\left(\alpha_{2}\right) \neq \emptyset$. Here it is possible to proceed recursively as above (applying the axioms (A1) and (A2)) and get all the cases, but they are many and the procedure is very long. There are the combs and all the cases listed in the families $\mathrm{x}, \mathrm{y}, \mathrm{z}, \mathrm{u}, \mathrm{v}, \mathrm{w}$ of the clan S. Alternatively, one can list all the cases by computer up to rank 9 . A colorconnected spherical system with $\Sigma=S$ of rank 9 is $b^{\mathrm{x}}(4,5), \mathrm{a}^{\mathrm{y}}(4,5), \mathrm{ab}^{\mathrm{y}}(4,5)$, $\mathrm{ad}^{\mathrm{y}}(4,5), \mathrm{bd}^{\mathrm{y}}(4,5)$ or a 9 -comb. Then it is possible to start from there to prove by induction that the only color-connected spherical systems with $\Sigma=S$ and rank $\geq 9$ (that are not combs) are:

- $\mathrm{bd}^{\mathrm{x}}(p-1, p), \mathrm{a}^{\mathrm{y}}(p-1, p), \mathrm{ab}^{\mathrm{y}}(p-1, p), \mathrm{ad}^{\mathrm{y}}(p-1, p), \mathrm{bd}^{\mathrm{y}}(p-1, p)$ (with odd rank $2 p-1)$, and

- $\mathrm{bd}^{\mathrm{x}}(p, p), \mathrm{a}^{\mathrm{y}}(p, p), \mathrm{ab}^{\mathrm{y}}(p, p), \mathrm{b}^{\mathrm{y}}(p, p), \mathrm{d}^{\mathrm{y}}(p, p), \mathrm{ad}^{\mathrm{y}}(p-1, p+1), \mathrm{a}^{\mathrm{y}}(2 p)$ (with even rank $2 p)$.

Plugs. Let us consider the following spherical systems:
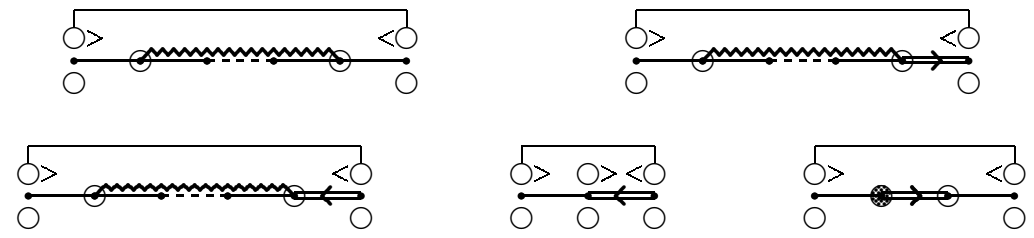

We say that two spherical roots $\sigma_{1}$ and $\sigma_{2}$ of a spherical $R$-system $\mathscr{S}$ lie in a plug if there exists $S^{\prime} \subset S$ with supp $\sigma_{1} \cup \operatorname{supp} \sigma_{2} \subset S^{\prime}$ such that the corresponding localization equals one of the above systems.

We easily obtain the following.

Lemma 2.19. A cuspidal spherical system (under the condition (2.1)) of rank $>2$ such that every pair of noncolor-adjacent spherical roots lies in a plug as above is primitive and is a member of the family $p$ of the clan $S$. 
Weakly-color-connected spherical systems. Let us abandon the condition (2.1). Notice that the following spherical system has the same rank, the same defect and the same Cartan pairing of the first spherical system of (2.2): it can thus be considered as a generalized plug, as well.

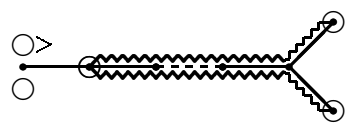

Further generalizing the notion of plugs, we define weak plugs: we say that two spherical roots $\sigma_{1}, \sigma_{2}$ of a spherical $R$-system $\mathscr{S}$ lie in a weak plug if there exists $S^{\prime} \subset S$ with supp $\sigma_{1} \cup \operatorname{supp} \sigma_{2} \subset S^{\prime}$ such that the corresponding localization satisfies the following:

$$
\Sigma^{\prime}=\left\{\sigma_{1}, \sigma_{2}, \sigma_{3}\right\} \text { and } \sigma_{1}, \sigma_{2} \text { are color-adjacent or have overlapping }
$$
support and there are two colors $D_{1}, D_{2}$ such that $D_{i} \in \Delta\left(\alpha_{i}\right)$ with $\alpha_{i} \in \operatorname{supp} \sigma_{3}$ and $c\left(D_{i}, \sigma_{i}\right) \neq 0$, for $i=1,2$.

Plugs are weak plugs. A particular weak plug is the following:

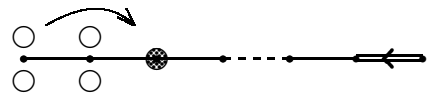

We say that two spherical roots are weakly-color-adjacent if they are coloradjacent or have overlapping support or lie in a weak plug; we say that two spherical roots are weakly-color-connected if they satisfy the same relation extended by transitivity; for short, we say that a spherical system is weakly-color-connected if its spherical roots are pairwise weakly-color-connected.

Lemma 2.20. A cuspidal weakly-color-connected spherical system

- is primitive and is a member of the clan $R$, of the clan $S$ (but not of the family q), or of the clan $T$,

- or has a tail of type $c(m)$,

- or has a positive $n$-comb: if we here assume in addition that $n=1$, then the positive 1-comb is primitive and the spherical system is a member of the family $p$.

Proof. Step 1. We keep on assuming color-connectedness but weaken condition (2.1) and assume that

if $\Delta(\alpha) \neq \emptyset$ and $\alpha \in \operatorname{supp} \sigma_{1} \cap \operatorname{supp} \sigma_{2}$, then at least one of the two spherical roots $\sigma_{1}, \sigma_{2}$ is of type $a(m)$ with $m>1$.

We must start with a spherical root of type $a(m), m>1$, and proceed recursively as above, but notice that now we are allowing only the following two further possibilities of pairs of color-adjacent spherical roots:
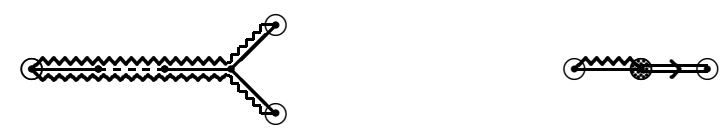

In particular, notice that in the following case the two spherical roots are not coloradjacent:

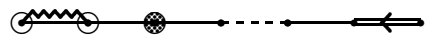


Only spherical roots of type $a(m)$ (with $m>1$ ) and $b(2)$ (with support not meeting $S^{p}$ ) are involved. We obtain only members of T-1 (of types A, B, D, E) and the case T-9.

Step 2. We allow plugs and generalized plugs (2.3). We obtain all the primitive spherical systems of the family $\mathrm{p}$ of the clan S (as in Lemma 2.19) and of the family a of the clan $\mathrm{T}$.

Step 3. We go on allowing the following as localizations
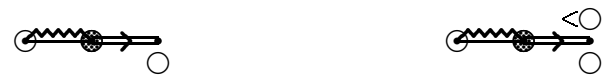

and we get the cases T-15, T-15 and T-16.

Allowing the following as localizations we get no new rank $>2$ cases (without positive $n$-combs with $n>1$ ).
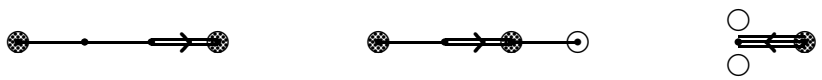

Step 4. We include all the pairs of spherical roots with overlapping support, namely also the following (with a root of type $c(m)$ with $m \geq 2$ ):
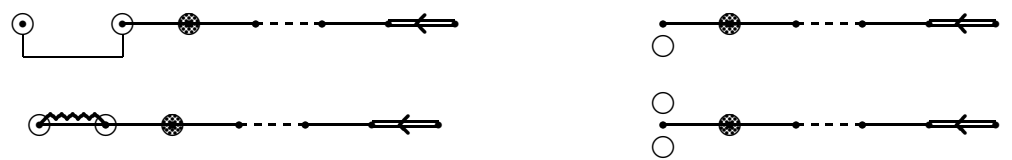

First, we get the cases T-17, T-18, T-19, ppc-1, ppc-2 and ppc-3. The rest are all cases (with a spherical root $\sigma$ of type $c(m)$ ) that can be constructed as in Section 2.4. These latter cases are many, but easy to construct, and most of them actually have a tail of type $c(m)$. In the notation of Section 2.4, they have a tail when the spherical system $\mathscr{S}^{\prime}$ has a homogeneous distinguished subset of colors that does not contain the color $D^{\prime}$ (and this can be checked case-by-case). The only cases without a tail are T-22 (if rank $>2$ ), T-25 and T-26.

Step 5. Finally, we include weak plugs and obtain the rest of the clan T (namely some members of $T-1$, the family $b$ and the rest of the family $c$ ) and the rest of the family $\mathrm{p}$ of spherical systems with a primitive positive 1-comb (namely only the case ppc-4).

Gluing. Let $\mathscr{S}=\left(S^{p}, \Sigma, A\right)$ be a cuspidal spherical $R$-system. Let $S^{\prime} \subset S$ be such that $\mathscr{S}^{\prime}=\left(\left(S^{\prime}\right)^{p}, \Sigma^{\prime}, A^{\prime}\right)$, the spherical system obtained from $\mathscr{S}$ by localization on $S^{\prime}$, is weakly-color-connected. Then $\mathscr{S}^{\prime}$ is called a weakly-color-connected component of $\mathscr{S}$ if $S^{\prime}$ is maximal with this property. The supports of the sets of spherical roots of the weakly-color-connected components are clearly disjoint, but not necessarily pairwise orthogonal. We will say that a cuspidal spherical $R$-system is obtained by gluing its weakly-color-connected components.

A spherical $R$-system is not uniquely determined by its weakly-color-connected components; see for example
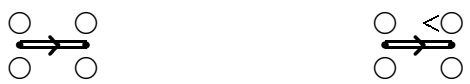
Remark 2.21. If $\mathscr{S}^{(1)}, \mathscr{S}^{(2)}$ are two weakly-color-connected components, let $\alpha_{1} \in$ $\Sigma^{(1)} \cap S^{(1)}$ and $\sigma_{2} \in \Sigma^{(2)}$ be not orthogonal. Then for $D \in A\left(\alpha_{1}\right)$ the value of $c\left(D, \sigma_{2}\right)$ is not always uniquely determined, but there are some constraints: if $D \in A\left(\alpha_{1}^{\prime}\right)$ for some $\alpha_{1}^{\prime} \in \Sigma^{(1)} \cap S^{(1)}$ different from $\alpha_{1}$ (we say that $D$ is not free), then $c\left(D, \sigma_{2}\right)=0$ or $\alpha_{1}^{\prime} \not \perp \sigma_{2}$ (notice that the latter does not necessarily give rise to a plug). In particular, if both colors $D^{+}, D^{-} \in A\left(\alpha_{1}\right)$ are not free, there can be no gluing with $\alpha_{1}$ not orthogonal to the spherical roots of other weakly-color-connected components.

Since we have classified all the cuspidal weakly-color-connected spherical systems, it is now possible to construct any cuspidal spherical system by gluing.

Erasable and quasi-erasable weakly-color-connected components. Let us introduce some further terminology: let $\mathscr{S}$ be a cuspidal spherical system. A weakly-colorconnected component $\mathscr{S}^{\prime}$ of $\mathscr{S}$ (or more generally a weakly-color-saturated localization $\mathscr{S}^{\prime}$ of $\mathscr{S}$, i.e. a localization of $\mathscr{S}$ obtained by gluing some of its weaklycolor-connected components) is called:

- isolated if $S^{\prime} \perp\left(S \backslash S^{\prime}\right)$;

- erasable if there exists a homogeneous good distinguished subset of colors $\Delta_{*}$ of $\mathscr{S}^{\prime}$ such that $c(D, \sigma)=0$, for all $D \in \Delta_{*}$ and all $\sigma \in \Sigma \backslash \Sigma^{\prime}$;

- quasi-erasable if there exists a nonempty good distinguished subset of colors $\Delta_{*}$ of $\mathscr{S}^{\prime}$ such that $c(D, \sigma)=0$, for all $D \in \Delta_{*}$ and all $\sigma \in \Sigma \backslash \Sigma^{\prime}$.

It directly follows that if a cuspidal spherical system $\mathscr{S}$ admits two weakly-colorconnected components such that one is isolated or both are quasi-erasable, then $\mathscr{S}$ is decomposable. This obviously generalizes to the case of two weakly-color-saturated localizations of $\mathscr{S}$ on disjoint sets $S^{(1)}, S^{(2)} \subset S$.

By abuse of terminology a weakly-color-connected component of rank 1 or 2 will be called a tail if it satisfies Definition 2.5] or 2.7 without the condition on the existence of the good distinguished subset of colors $\Delta^{\prime}$. We will see below (Lemma 2.22) that in these hypotheses such a $\Delta^{\prime}$ always exists.

We now analyze all the cuspidal weakly-color-connected spherical systems which have been classified above. Case-by-case we start with a spherical system $\mathscr{S}^{(1)}$, see how it can be glued to another spherical system $\mathscr{S}^{(2)}$ such that $\mathscr{S}^{(1)}$ and $\mathscr{S}^{(2)}$ are weakly-color-saturated and say whether $\mathscr{S}^{(1)}$ is necessarily isolated, erasable or quasi-erasable.

Rank 1. If a weakly-color-connected component is of rank 1 and equal to

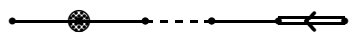

then it is necessarily isolated. The same is true if the spherical root of the weaklycolor-connected component has support of type $F_{4}$ or $G_{2}$. If it is equal to

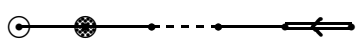

then it is necessarily erasable. If it is equal to the following case with a spherical root of type $b(m), m \geq 3$

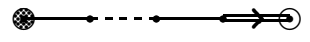

then it is necessarily quasi-erasable. If its spherical root is of type $b(m), m \geq 1$ $\left(\alpha_{n} \in S^{p}\right.$ if $\left.m \geq 2\right), 2 b(m), m \geq 1$ or $d(m), m \geq 3$, then it is a tail. If its spherical 
root is of type $a a$, then it is a tail or it is glued to an $n$-comb component; if $n=1$ we get a rank 2 case (with support of type $C_{3}$ ) which is isolated. If it is equal to

then it is isolated or it is glued to an $n$-comb component; if $n=1$ we get a rank 2 case which is isolated. If it is equal to the case of a spherical root of type $b(2)$ whose support does not meet $S^{p}$, then it is quasi-erasable or it is glued to two comb components so that the whole spherical system is decomposable. Notice that it remains the case of a spherical root of type $a(m), m \geq 1$, which will occur in some primitive cases below.

Rank 2. Analogously, if a weakly-color-connected component is of rank 2, then it is either isolated or erasable or quasi-erasable

or a tail or glued to an $n$-comb component (the corresponding cases are erasable; if $n=1$ and the 1 -comb is primitive, they are rank 3 spherical systems: ppc-5, ppc-6. ppc-7. ppc-8, ppc-9) or a 2-comb or with two spherical roots of type $a(m), m \geq 2$ with overlapping supports (the two latter cases will be analyzed below).

Clan $R$. Let us start with $\mathrm{a}^{\text {aa }}(p, p), p>2$. It can only be glued to an $n$-comb component; if $n=1$ we get the case ppc-5, which is isolated. Starting with $\mathrm{a}^{2 \mathrm{a}}(p)$ the situation is very similar: we get the case ppc-6, which is isolated. Analogously with the case R-9 we get the case ppc-7, which is isolated. The cases R-2, R-11. $\mathrm{R}-12$ and R-13 are erasable. All the other cases of the clan R are isolated.

Clan S. Let us consider the color-connected cases of the clan S. A weakly-colorconnected component of this kind is isolated or erasable or it is equal to one of the following cases:

- $\operatorname{bd}^{\times}(p, p)$ is quasi-erasable or glued to combs in a decomposable system;

- $\operatorname{bd}^{\times}(p-1, p)$ is quasi-erasable or, if $p=3$ glued to a comb and, if the latter is primitive, as in ppc-11, which is isolated or, if $p=2$ namely $\mathrm{a}^{\times}(1,1,1)$, not necessarily quasi-erasable;

- $\mathrm{a}^{\mathrm{y}}(p, p)$ is erasable or glued to combs in a decomposable system or, if $p=2$, is not necessarily quasi-erasable;

- $\mathrm{a}^{\mathrm{y}}(p-1, p)$ is quasi-erasable or glued to combs in a decomposable system or, if $p=2$, glued to a comb as in ppc-12, which is isolated;

- $\mathrm{ab}^{\mathrm{y}}(p, p)$ and $\mathrm{ad}^{\mathrm{y}}(p+1, p+1)$ are erasable or glued to combs and, if the latter are primitive, as in ppc-13, ppc-14. ppc-15 or ppc-16, which are erasable;

- $\mathrm{ab}^{\mathrm{y}}(p-1, p), \mathrm{ad}^{\mathrm{y}}(p-1, p+1)$ and $\mathrm{a}^{\mathrm{z}}(1,3)$ are quasi-erasable;

- $\mathrm{a}^{\mathrm{u}}(5)$ is quasi-erasable;

- $a^{z}(1,3)$ and $b^{w}(3)$ can only be glued to combs in a decomposable system.

A weakly-color-connected component, if equal to a remaining case of the clan S, is isolated or erasable or is equal to S-62, S-68. S-75 (of rank 5) or S-76 (of rank 4) which are quasi-erasable, or can only be glued to combs in a decomposable system.

Clan $T$. Let us consider the cases of the clan $T$ with only spherical roots of type $a(m)$. A weakly-color-connected component of this kind is isolated or erasable or it is equal to one of the following cases:

- $\mathrm{a}^{\mathrm{m}}(2 p+1)$ is erasable or glued to combs in a decomposable system or, if $p=2$, is not necessarily quasi-erasable;

- $\mathrm{a}^{\mathrm{m}}(2 p+2)$ is quasi-erasable or glued to combs in a decomposable system; 
- $\mathrm{d}^{\mathrm{m}}(2 p+1)$ and $\mathrm{e}^{\mathrm{m}}(6)$ are quasi-erasable;

- T-2 and T-10 are quasi-erasable;

- T-9 is not necessarily quasi-erasable.

A weakly-color-connected component, if equal to a remaining case of the clan $\mathrm{T}$, is isolated or erasable or is equal to $\mathrm{b}^{\mathrm{m}}(2 p+2)$, $\mathrm{T}-15$ or $\mathrm{T}-15$, which are quasierasable, or can only be glued to combs in a decomposable system.

Tails of type $c(m)$. For completeness we have to also consider weakly-colorconnected spherical systems with a tail of type $c(m)$ (with overlapping support), but as components they are necessarily isolated, erasable or glued to combs in a decomposable system. This can be seen by "collapsing" the spherical root of type $c(m)$ (as in Section 2.3 for the cases of the family c): one obtains only weakly-colorconnected spherical systems that have already been considered above.

Remaining cases. An $n$-comb that is a weakly-color-connected component by itself can be glued to a positive 1-comb (cases ppc-9 and ppc-10 which are erasable) or more generally to a spherical system with only spherical roots of type $a(1)$ and a free color (some cases listed in the family q of the clan $S$ which are erasable).

Finally, if we have a rank 1 weakly-color-connected component with a spherical root $\sigma$ of type $a(m)$, denote by $D_{1}, D_{2}$ its colors, such that there exist two weaklycolor-connected spherical roots $\sigma_{1}, \sigma_{2} \neq \sigma$ with $c\left(D_{i}, \sigma_{i}\right) \neq 0, i=1,2$, then we obtain only the remaining cases listed in the family $\mathrm{q}$ of the clan $\mathrm{S}$ which are erasable.

Type A connected subdiagrams of the Dynkin diagram.

Lemma 2.22. Let $S^{(1)} \sqcup S^{(2)}=S$ be such that the corresponding localizations $\mathscr{S}^{(1)}, \mathscr{S}^{(2)}$ of $\mathscr{S}$ are weakly-color-saturated. Assume that the Dynkin diagram of $S^{(1)}$ has a connected component of type $\mathrm{A}_{r}$, and let $\alpha_{1}, \ldots, \alpha_{r}$ be its simple roots (labelled as usual). Then

(1) if $S^{(1)} \backslash\left\{\alpha_{1}, \alpha_{r}\right\}$ is orthogonal to $S^{(2)}$, then $\alpha_{1}+\ldots+\alpha_{r} \in \Sigma$ (and the localization of $\mathscr{S}$ on $\left\{\alpha_{1}, \ldots, \alpha_{r}\right\}$ is a weakly-color-connected component by itself) or $\mathscr{S}^{(1)}$ is quasi-erasable;

(2) if $S^{(1)} \backslash\left\{\alpha_{1}\right\}$ is orthogonal to $S^{(2)}$, then $\mathscr{S}^{(1)}$ is erasable.

Proof.

(1) It is enough to look at the weakly-color-connected spherical systems of rank $>1$ that are not necessarily quasi-erasable: notice that they are quasierasable if their support meets a connected component of type A of the Dynkin diagram of $S$.

(2) Similarly, it is enough to look at the weakly-color-connected spherical systems that are not necessarily erasable: notice that they are erasable if their support contains an extremal simple root of a connected component of type A of the Dynkin diagram of $S$.

The following completes the proof of Theorems 2.10 and 2.12

Proposition 2.23. Let $\mathscr{S}$ be a cuspidal spherical $R$-system without tails and positive $n$-combs with $n>1$. If $\mathscr{S}$ is not primitive and has no primitive positive 1-combs, there exists a decomposition $S^{(1)} \sqcup S^{(2)}=S$ such that the corresponding localizations $\mathscr{S}^{(1)}, \mathscr{S}^{(2)}$ of $\mathscr{S}$ are weakly-color-saturated and both quasi-erasable; therefore $\mathscr{S}$ is decomposable. 
Proof. We are left to consider the cuspidal spherical $R$-systems $\mathscr{S}$, without tails and positive $n$-combs with $n>1$, with a weakly-color-connected component that is not quasi-erasable. A case-by-case analysis of the weakly-color-connected spherical systems that are not necessarily quasi-erasable, together with Lemma 2.22 shows that, if $\mathscr{S}$ is not primitive and has no primitive positive 1-combs, we can always choose such a decomposition $S^{(1)} \sqcup S^{(2)}=S$.

Furthermore, another direct consequence of Lemma 2.22 is the following, which applies more generally to all spherical systems.

Proposition 2.24. Let $\mathscr{S}$ be a spherical R-system. Then there exists a decomposition $S^{(1)} \sqcup S^{(2)}=S$ such that the corresponding localization $\mathscr{S}^{(1)}$ is weakly-colorsaturated and erasable.

Proof. Notice that for every decomposition $S^{(1)} \sqcup S^{(2)}=S$ either $S^{(1)} \perp S^{(2)}$ or the Dynkin diagram of $S^{(1)}$ (or of $S^{(2)}$ ) has a connected component of type A. Therefore, by Lemma 2.22, for all spherical $R$-systems $\mathscr{S}$ there exists such a decomposition.

Therefore, by the way, we have also proved the following.

Corollary 2.25. The whole set of colors of a spherical system is distinguished and good.

Proof. Let $S^{(1)} \sqcup S^{(2)}=S$ be a decomposition as in Proposition 2.24, Call $\Delta_{*}^{(1)}$ a corresponding homogeneous distinguished subset of colors of $\mathscr{S}^{(1)}$. It can be identified with a good distinguished subset of colors of $\mathscr{S}$. Proposition 2.24 can then be applied recursively to the quotient $\mathscr{S} / \Delta_{*}^{(1)}$ (which is equal to $\mathscr{S}^{(2)}$ up to induction).

\section{Quotients of GENERAL SPHERICAL SYSTEMS}

Theorem 3.1. All distinguished subsets of colors of a spherical system are good.

First, let us show that without loss of generality we can assume that $\Sigma \cap \Sigma / \Delta_{*}=$ $\emptyset$. Let $\mathscr{S}=\left(S^{p}, \Sigma, A\right)$ be a spherical $R$-system with set of colors $\Delta$ and a distinguished subset $\Delta_{*} \subset \Delta$. Let $\sigma \in \Sigma \cap \Sigma / \Delta_{*}$. Then for all $D \in \Delta_{*}$ one has $c(D, \sigma)=0$ : for all $\alpha \in S, D \in \Delta_{*} \cap \Delta(\alpha)$ implies that $\alpha \perp \operatorname{supp} \sigma$ or $\alpha \in \Sigma$ with $D \in A(\alpha)$ and $A(\alpha) \backslash \Delta_{*} \neq \emptyset$. Therefore, the subset $\Delta_{*}$ of colors of $\mathscr{S}$ is good provided it is good as subset of colors of the spherical $R$-system $\left(S^{p}, \Sigma \backslash\{\sigma\}, \bigcup_{\alpha \neq \sigma} A(\alpha)\right)$.

Moreover, it is clearly enough to prove the theorem for the minimal distinguished subsets. Recall that all the minimal distinguished subsets of the rank $\leq 2$ spherical systems or of the primitive spherical systems or of the spherical systems with a primitive positive 1-comb are good (Remarks 1.13 and 2.13). The same is true for all the weakly-color-connected spherical systems. Indeed, recall that the systems with a tail of type $c(m)$ (with overlapping support) behave as their analogues obtained by "collapsing" the tail (as in Section 2.3 for the cases of the family c); see Section 2.5 for the notion of weak-color-connectedness. 
Other spherical systems with a minimal distinguished subset of colors $\Delta_{*}$ with $\Sigma \cap \Sigma / \Delta_{*}=\emptyset$ are the following:
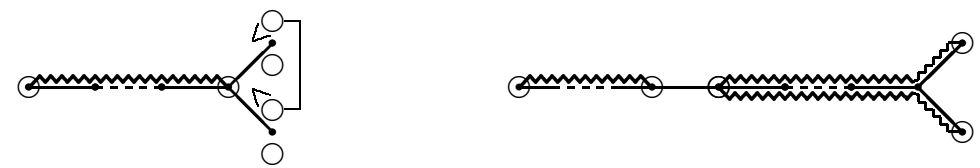

We prove the theorem by showing that no other spherical system admits a minimal distinguished subset of colors $\Delta_{*}$ with $\Sigma \cap \Sigma / \Delta_{*}=\emptyset$.

Let $\mathscr{S}=\left(S^{p}, \Sigma, A\right)$ be a spherical $R$-system with set of colors $\Delta$ and a minimal distinguished subset $\Delta_{*} \subset \Delta$. Let $\mathscr{S}^{(1)}, \mathscr{S}^{(2)}$ be weakly-color-saturated localizations of $\mathscr{S}$ corresponding to $S^{(1)} \sqcup S^{(2)}=S$.

The minimality of $\Delta_{*}$ implies the following: if $\Delta_{*} \not \subset \Delta^{(1)}$, then every nonempty subset of $\Delta_{*} \cap \Delta^{(1)}$ that is distinguished in $\mathscr{S}^{(1)}$ must contain a color $D$ such that $c(D, \sigma)<0$ for some $\sigma \in \Sigma^{(2)}$.

We will say that $D \in \Delta_{*} \cap \Delta^{(1)}$ and $\sigma \in \Sigma^{(2)}$ such that $c(D, \sigma)<0$ are in gluing relation.

Moreover, $\Delta_{*}$ is distinguished in $\mathscr{S}$. Therefore, assuming that $\Delta_{*} \cap \Delta^{(2)}$ is good distinguished in $\mathscr{S}^{(2)}$, if $\sigma \in \Sigma^{(2)}$ is in gluing relation with some $D \in \Delta_{*} \cap \Delta^{(1)}$, then there exists an element of $\mathbb{N}\left(\Delta_{*} \cap \Delta^{(2)}\right)$ that is positive on $\Sigma^{(2)}$ and strictly positive on $\sigma$.

Clearly, by symmetry, the above terminology and implications are also valid if we exchange the role of the indices 1 and 2 .

Lemma 3.2. Let $\mathscr{S}$ be a nonweakly-color-connected spherical $R$-system with a minimal distinguished subset of colors $\Delta_{*}$. Assume that there is no proper subset $S^{\prime}$ with weakly-color-saturated localization $\mathscr{S}^{\prime}$ such that $\Delta_{*} \not \subset \Delta^{\prime}$. Then there exist nonempty subsets $S^{(1)}, S^{(2)}$ with $S^{(1)} \sqcup S^{(2)}=S$ and weakly-color-saturated $\mathscr{S}^{(1)}, \mathscr{S}^{(2)}$ localizations with a unique simple root $\alpha \in S^{(1)}$ nonorthogonal to $S^{(2)}$.

Proof. First, we can assume that all weakly-color-connected components satisfying

at least two connected components of the support are nonorthogonal to their complement

have a nonfree color that is in gluing relation with some spherical root of other weakly-color-connected components. Indeed, it can be checked case-by-case that a cuspidal weakly-color-connected spherical system, that as a weakly-color-connected component of a bigger spherical system can satisfy the condition (3.2), has no distinguished subset $\Delta_{*}$ such that

- every minimal distinguished subset of $\Delta_{*}$ contains a free color and

- there exists a positive element of $\mathbb{N} \Delta_{*}$ that is strictly positive on some spherical root.

A nonfree color $D$ can be in gluing relation with only one spherical root $\sigma$ of other weakly-color-connected components. If there is such a color $D$, cut the Dynkin diagram between the support of $\sigma$ and $\alpha$ for all $\alpha \in S$ such that $D \in \Delta(\alpha)$. Define $S^{(1)}$ as the minimal subset of $S$ containing the connected component of the Dynkin diagram (after the cut prescribed above) that contains supp $\sigma$ and such that $\mathscr{S}^{(1)}$ is weakly-color-saturated. By the above assumption, $S^{(1)}$ and its complement enjoy the required properties. 
We are left with the case when there is no weakly-color-connected component satisfying the condition (3.2). Here there always exists a weakly-color-connected component whose support $S^{(1)}$ contains only one simple root that is nonorthogonal to $S \backslash S^{(1)}$.

As a consequence, up to exchanging the role of $S^{(1)}$ and $S^{(2)}$ in the above lemma, we are in the following situation.

Lemma 3.3. In the hypothesis of Lemma 3.2, denote by $\alpha^{(1)}$ the unique simple root in $S^{(1)}$ nonorthogonal to $S^{(2)}$.

Then there exist

- $D^{(1)} \in \Delta_{*} \cap \Delta^{(1)}\left(\alpha^{(1)}\right)$ and $\sigma^{(1)} \in \Sigma^{(1)}$ with $\alpha^{(1)} \in \operatorname{supp} \sigma^{(1)}$ (hence $\left.c\left(D^{(1)}, \sigma^{(1)}\right) \geq 0\right)$,

- $D^{(2)} \in \Delta^{(2)}(\alpha)$, for all $\alpha \in S^{(2)}$ nonorthogonal to $\alpha^{(1)}$, and $\sigma_{k}^{(2)} \in \Sigma^{(2)}$, for $k=1, \ldots, n$ and $1 \leq n \leq 3$, with $\operatorname{supp} \sigma_{k}^{(2)}$ nonorthogonal to $\alpha^{(1)}$ (hence $c\left(D^{(2)}, \sigma_{k}^{(2)}\right) \geq 0$ for all $\left.k\right)$,

such that

$$
\begin{aligned}
& D \in \Delta_{*} \cap \Delta^{(i)} \text { and } \sigma \in \Sigma^{(j)} \text { with } i \neq j \text { are in gluing relation only } \\
& \text { if } D=D^{(1)} \text { and } \sigma=\sigma_{k}^{(2)} \text {, or } D=D^{(2)} \text { and } \sigma=\sigma^{(1)} \text {. }
\end{aligned}
$$

Under this condition we conclude the proof.

First, assume that $n=1$ and set $\sigma^{(2)}=\sigma_{1}^{(2)}$. We claim that

(a) $D^{(1)}$ and $D^{(2)}$ are positive on $\Sigma^{(1)}$ and $\Sigma^{(2)}$, respectively, or

(b) there exist $i$ (equal to 1 or 2) and a spherical root $\sigma \neq \sigma^{(i)}$ in $\Sigma^{(i)}$ with $c\left(D^{(i)}, \sigma\right)>0$ (if $c\left(D^{(i)}, \sigma^{(i)}\right)>0$ this means that $D^{(i)}$ is not free).

Indeed, by the list of nonweakly-color-connected rank 2 spherical systems it follows that $c\left(D^{(1)}+D^{(2)}, \sigma^{(i)}\right) \leq 0$. Let $\tilde{D}=\sum n_{D} D$ be a positive element of $\mathbb{N}_{>0} \Delta_{*}$. Set $\tilde{D}^{(i)}=\sum n_{D} D$ for all $D \in \Delta_{*} \cap \Delta^{(i)} \backslash\left\{D^{(i)}\right\}$. Notice that $\Delta_{*} \cap \Delta^{(i)}=$ $\left\{D^{(i)}\right\}$ if and only if $D^{(i)}$ is positive on $\Sigma^{(i)}$. If there exists no $\sigma \neq \sigma^{(i)}$ in $\Sigma^{(i)}$ with $c\left(D^{(i)}, \sigma\right)>0$, then $\tilde{D}^{(i)}$ is positive on $\Sigma^{(i)} \backslash\left\{\sigma^{(i)}\right\}$. If for both $i=1,2$ there exists no $\sigma \neq \sigma^{(i)}$ with $c\left(D^{(i)}, \sigma\right)>0$, then $c\left(\tilde{D}^{(i)}, \sigma^{(i)}\right) \geq 0$ for $i=1,2$. Therefore, under the latter condition, if (for $i$ equal to 1 or 2 ) $D^{(i)}$ is not positive on $\Sigma^{(i)}$, then $\Delta_{*} \cap \Delta^{(i)} \backslash\left\{D^{(i)}\right\}$ is not empty and $\tilde{D}^{(i)}$ is positive on $\Sigma^{(i)}$, a contradiction.

(a) If the two colors $D^{(1)}$ and $D^{(2)}$ are positive on $\Sigma^{(1)}$ and $\Sigma^{(2)}$, respectively, namely $\Delta_{*}=\left\{D^{(1)}, D^{(2)}\right\}$ and $c\left(D^{(1)}+D^{(2)}, \sigma^{(i)}\right)=0$, it is enough to look at the rank 2 case with spherical roots $\sigma^{(1)}$ and $\sigma^{(2)}$.

(b) If there exists $\sigma \neq \sigma^{(1)}$ in $\Sigma^{(1)}$ with $c\left(D^{(1)}, \sigma\right)>0$, then $\sigma^{(1)}$ would be of type $a(m)$, but in this case $\sigma^{(1)}$ and $\sigma^{(2)}$ would be weakly-color-connected (contradiction).

Now assume $n=2$. Then the spherical roots $\sigma^{(1)}, \sigma_{1}^{(2)}$ and $\sigma_{2}^{(2)}$ are of type $a$; moreover, the spherical roots $\sigma_{1}^{(2)}$ and $\sigma_{2}^{(2)}$ are color-adjacent or have overlapping supports. Locally, they are as in (3.1) or in ppc-9. Analogously as above, $D^{(1)}$ and $D^{(2)}$ are positive on $\Sigma^{(1)}$ and $\Sigma^{(2)}$, respectively: indeed, if $D^{(2)}$ is not positive, then there exists a distinguished subset of $\Delta_{*} \cap \Delta^{(2)} \backslash\left\{D^{(2)}\right\}$. Therefore, $\Delta_{*}=$ $\left\{D^{(1)}, D^{(2)}\right\}$.

Finally, if $n=3$, the only possible case is ppc-10. 


\section{APPEndiX: SPheriCAL SySTEMS OF RANK $\leq 2$}

Let $S_{i}, i=1,2$, be subsets of $S$ with $S_{1} \sqcup S_{2}=S$ and $S_{1} \perp S_{2}$. Let $\mathscr{S}_{i}=$ $\left(S_{i}^{p}, \Sigma_{i}, A_{i}\right), i=1,2$, be spherical $R_{i}$-systems. The direct product of $\mathscr{S}_{1}$ and $\mathscr{S}_{2}$ is the spherical $R$-system $\left(S_{1}^{p} \sqcup S_{2}^{p}, \Sigma_{1} \sqcup \Sigma_{2}, A_{1} \sqcup A_{2}\right)$ where $c(D, \sigma)=0$ for all $D \in A_{i}$, $\sigma \in \Sigma_{j}$ and $i \neq j$.

Here we give the Luna diagrams of all the cuspidal spherical systems of rank 1 and of all the cuspidal spherical systems of rank 2 that are not a direct product of two spherical systems of rank 1, together with their minimal quotient spherical systems.

For the cuspidal spherical $R$-systems of rank $1, n$ denotes the rank of $R$.

For the cuspidal spherical systems of rank 2 that are not a direct product of two spherical systems of rank $1, p$ and $q$ denote the rank of the supports of the two spherical roots: we use identifications between spherical roots for small $p$ and $q$ as stated in Definition 1.1

Rank 1.

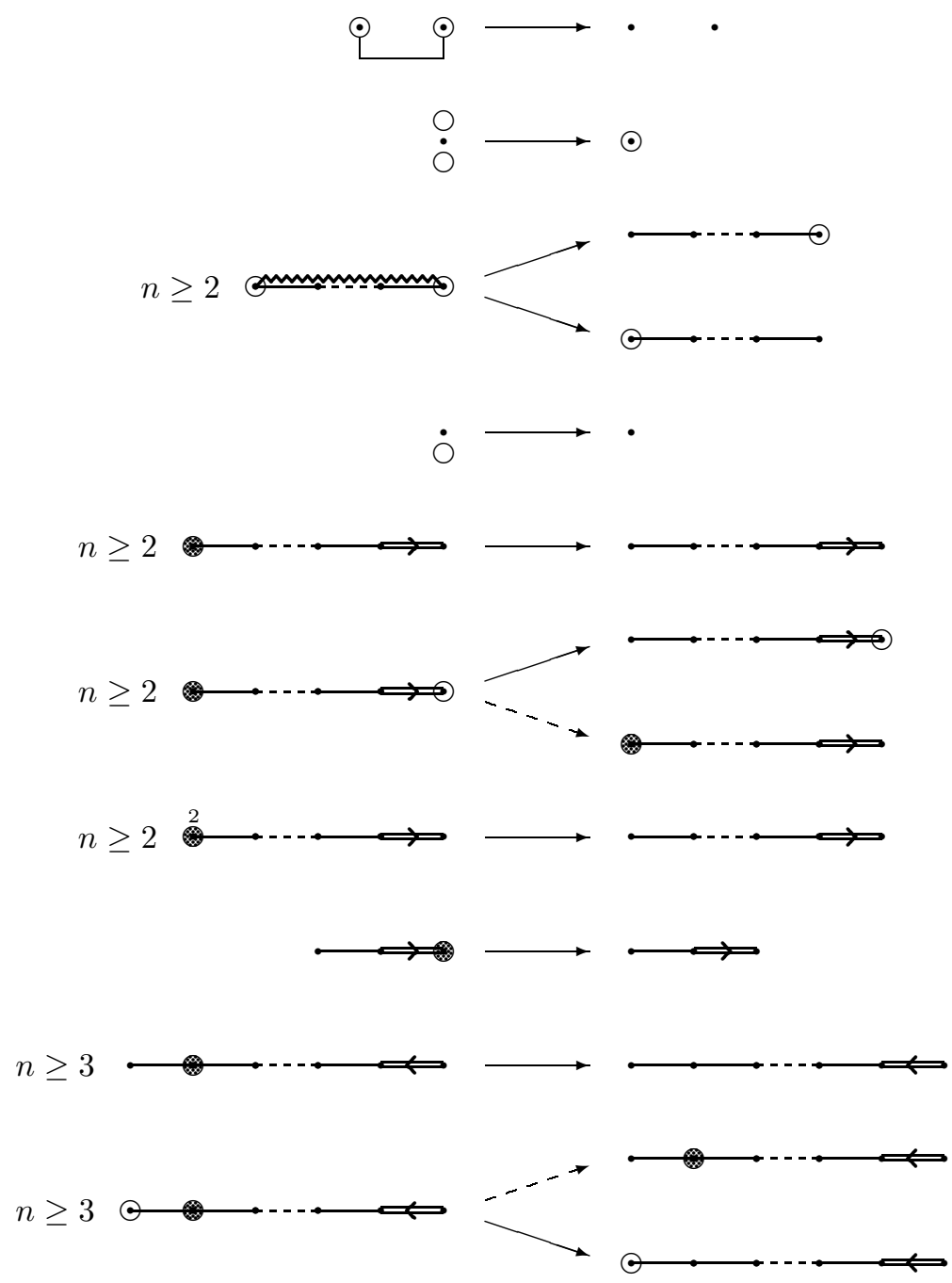




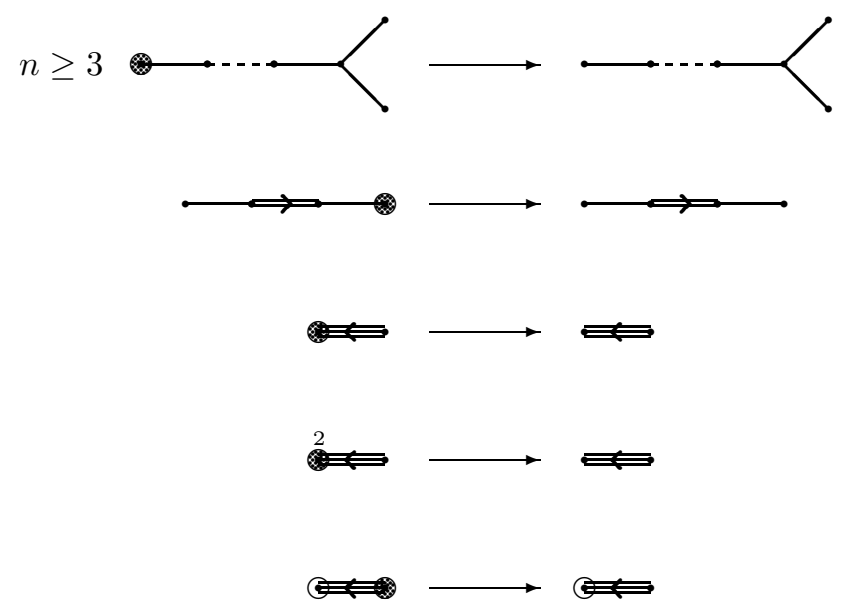

Rank 2.

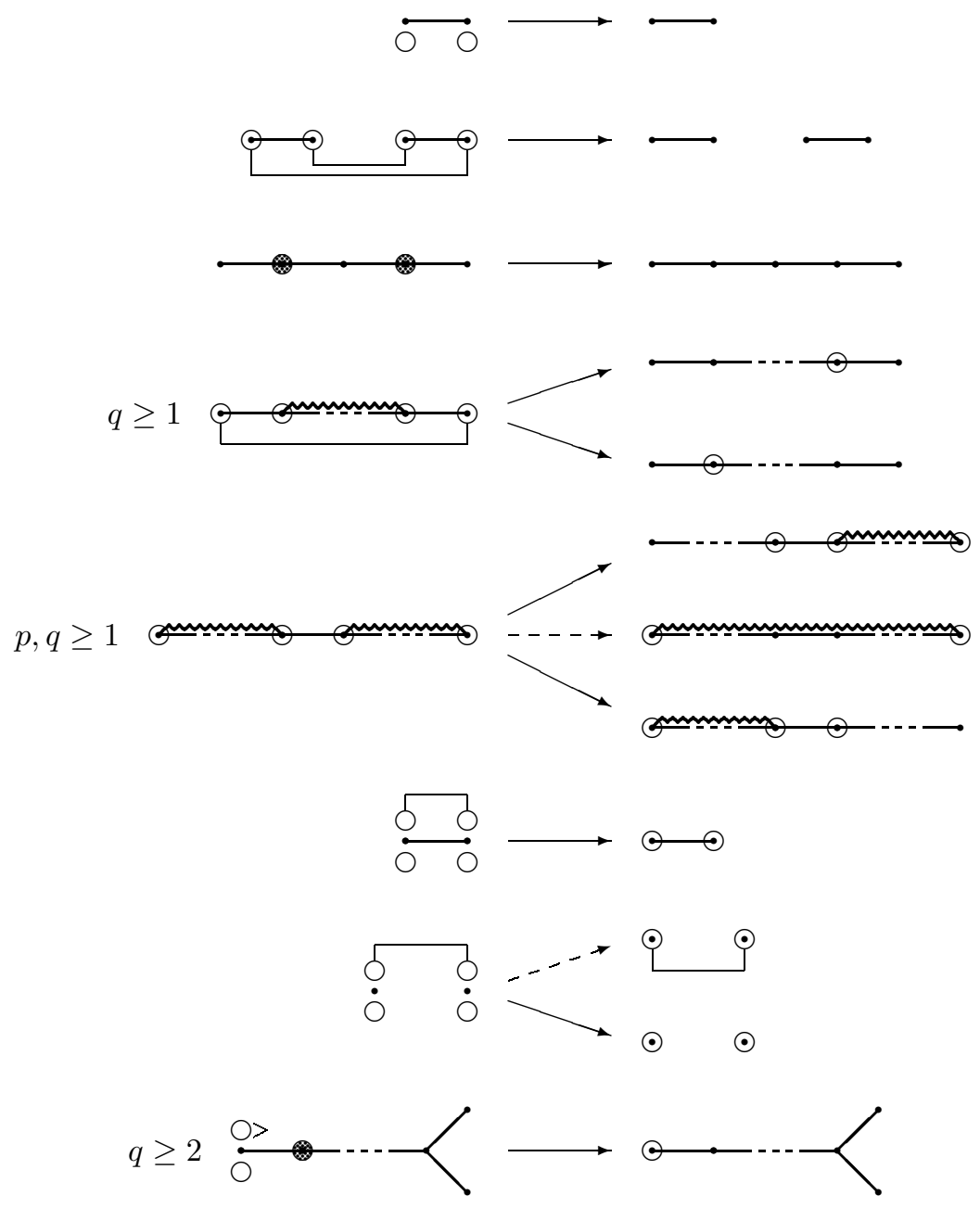




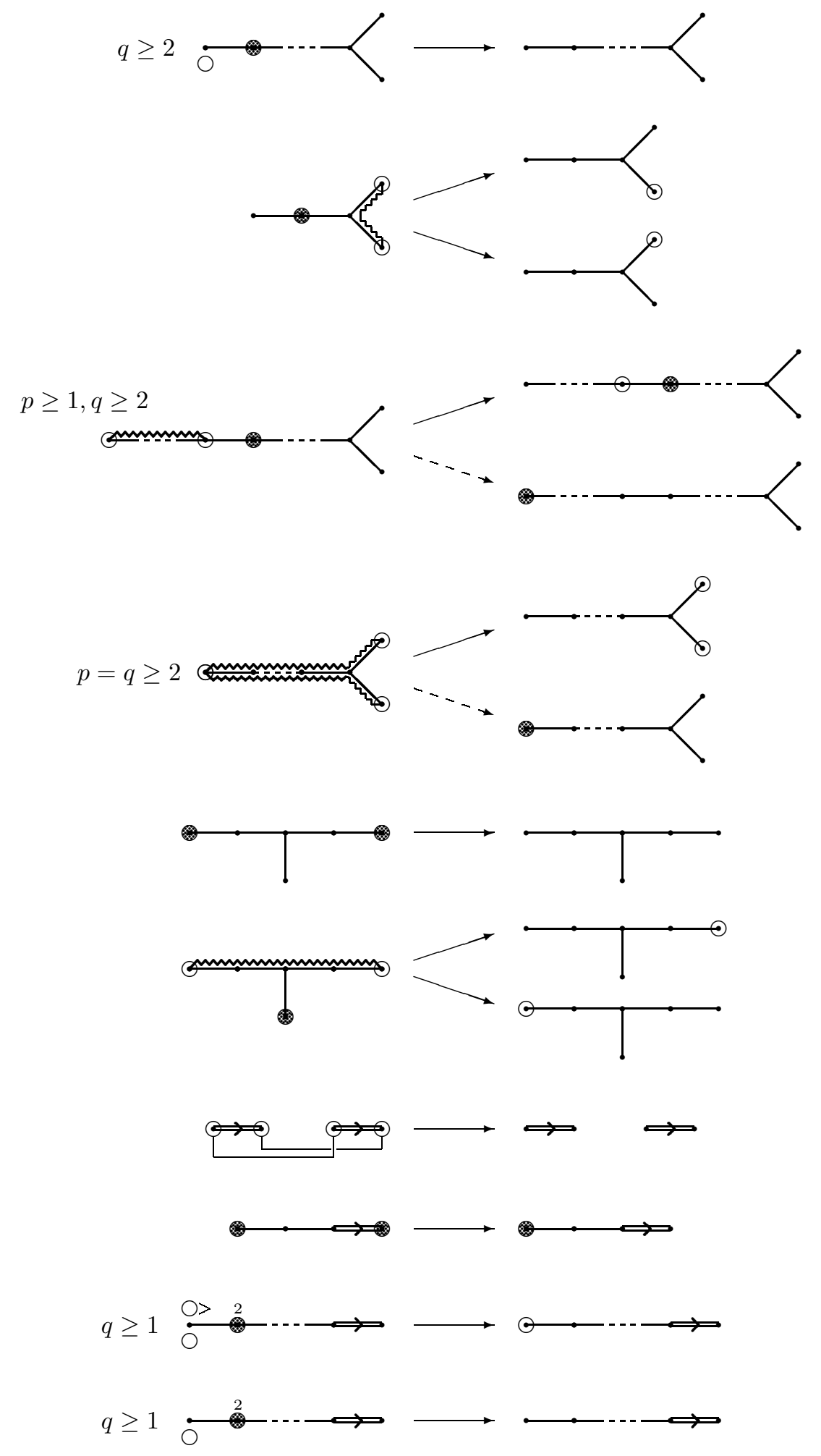




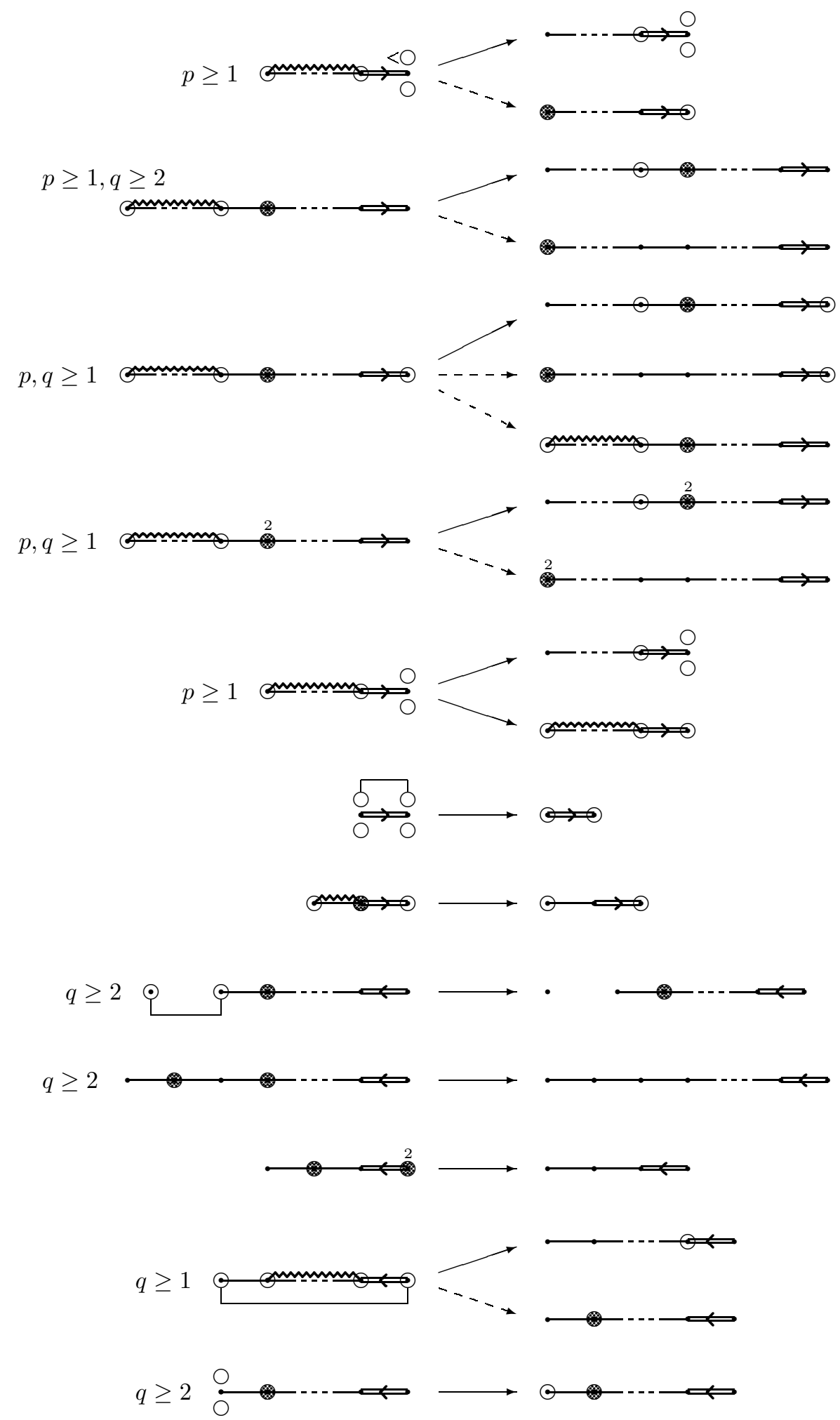




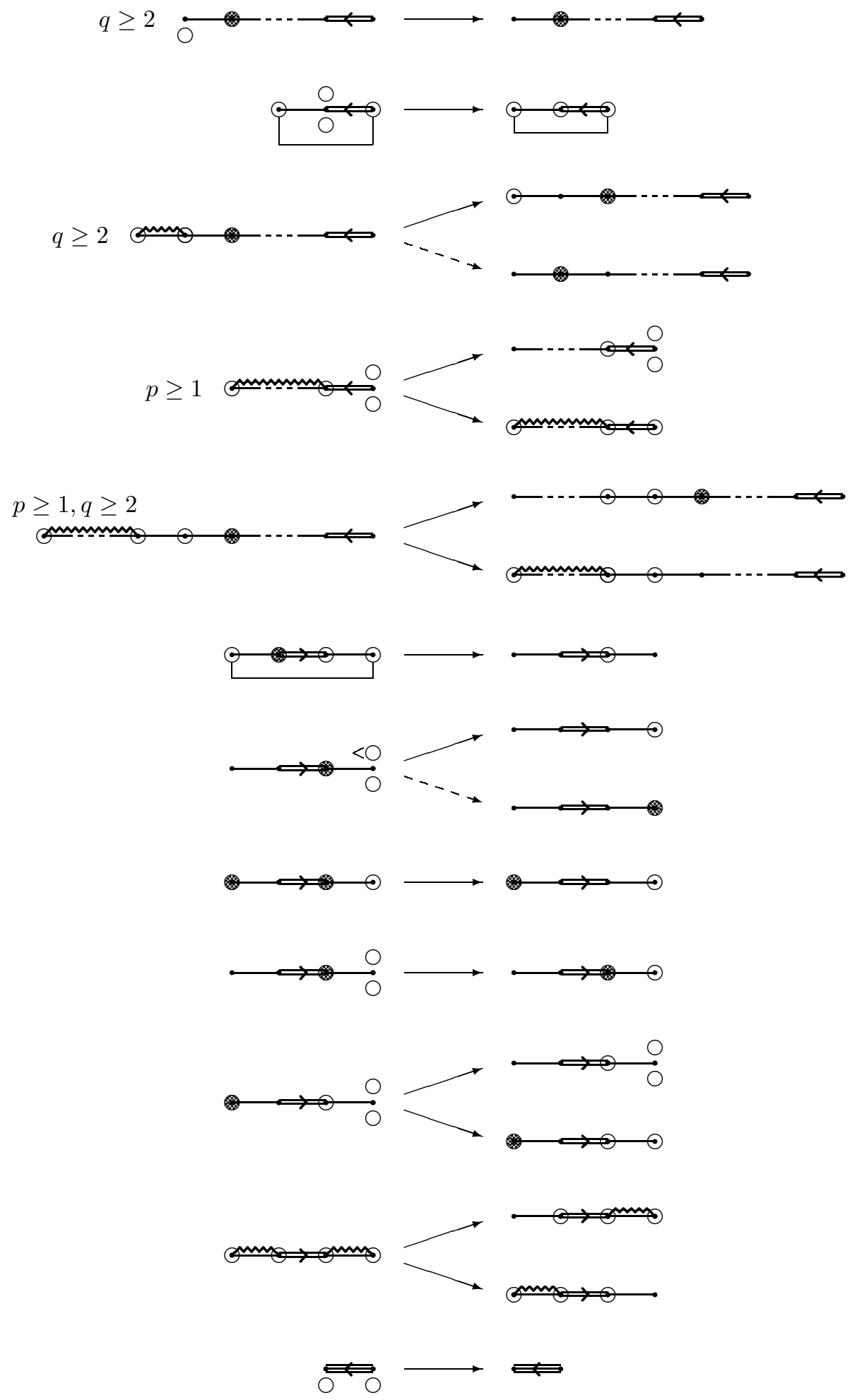




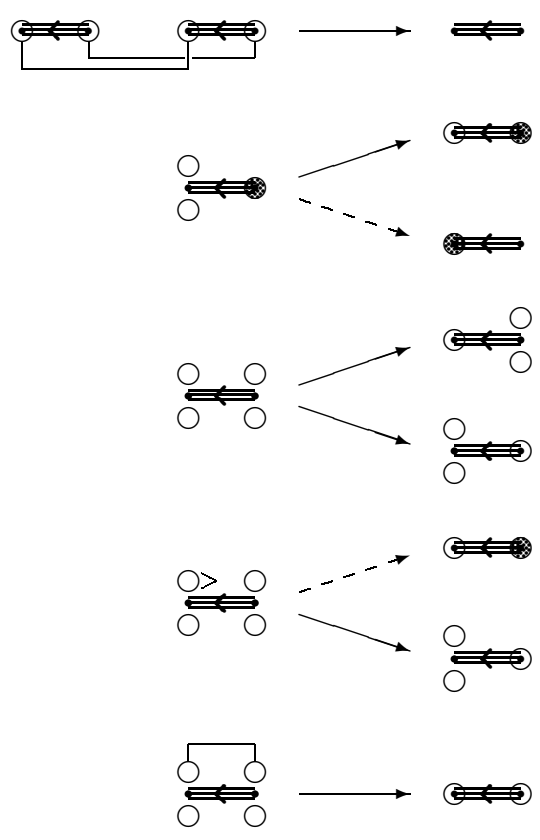

\section{REFERENCES}

[Br07] P. Bravi, Wonderful varieties of type E, Representation Theory, 11 (2007), 174-191. MR2346359 (2008h:14046)

[BCF08] P. Bravi, S. Cupit-Foutou, Equivariant deformations of the affine multicone over a flag variety, Adv. Math. 217 (2008), 2800-2821. MR2397467 (2009a:14061)

[BCF10] P. Bravi, S. Cupit-Foutou, Classification of strict wonderful varieties, Ann. Inst. Fourier (Grenoble) 60 (2010), 641-681. MR2667789

[BL11] P. Bravi, D. Luna, An introduction to wonderful varieties with many examples of type $F_{4}$, J. Algebra 329 (2010), 4-51. MR2769314

[BP05] P. Bravi, G. Pezzini, Wonderful varieties of type D, Represent. Theory, 9 (2005), 578637. MR2183057 (2006g:14078)

[BP11] P. Bravi, G. Pezzini, A constructive approach to the classification of wonderful varieties, arXiv:1103.0380v1 .

[Bo] N. Bourbaki, Éléments de mathématique. Groupes et Algèbres de Lie. Chapitre IV: Groupes de Coxeter et systèmes de Tits. Chapitre V: Groupes engendrés par des réflexions. Chapitre VI: Systèmes de racines. Actualités Scientifiques et Industrielles, No. 1337, Hermann, Paris, 1968. MR0240238 (39:1590)

[CF08] S. Cupit-Foutou, Invariant Hilbert schemes and wonderful varieties, arXiv:0811.1567v2 .

[CF09] S. Cupit-Foutou, Wonderful varieties: a geometrical realization, arXiv:0907.2852v3 .

[Lo09] I.V. Losev, Uniqueness property for spherical homogeneous spaces, Duke Math. J. 147 No. 2 (2009), 315-343. MR2495078 (2010c:14055)

[Lu01] D. Luna, Variétés sphériques de type A, Publ. Math. Inst. Hautes Études Sci. 94 (2001), 161-226. MR:1896179 (2003f:14056)

[P03] G. Pezzini, Wonderful varieties of type $C$, Ph.D. Thesis, Dipartimento di Matematica, Università La Sapienza, Rome, 2003.

[W96] B. Wasserman, Wonderful varieties of rank two, Transform. Groups 1 (1996), no. 4, 375-403. MR.1424449 (97k:14051) ITALY

Dipartimento di Matematica, Università La Sapienza, P.le A. Moro 5, 00185 Roma,

E-mail address: bravi@mat.uniroma1.it 